\title{
MEMORIAL TRIBUTE
}

\section{Jean-Marc Fontaine (1944-2019)}

\section{Pierre Berthelot, Luc Illusie, Nicholas M. Katz, William Messing, and Peter Scholze}

Jean-Marc Fontaine passed away on January 29, 2019. For half a century, and up until his very last days, he devoted his immense talent and energy to the development of one of the most powerful tools now available in number theory and arithmetic geometry, p-adic Hodge theory. His work has had and will continue to have a tremendous influence. It has many deep applications. With the following texts, we would like to express our admiration, gratitude, and friendship.

Jean-Marc was born on March 13, 1944, in BoulogneBillancourt, a city located in the southwest suburbs of Paris. Boulogne-Billancourt was then mainly known as hosting one of the biggest car plants in France, and as a labor union stronghold. However, Jean-Marc's family background was different. Close to the Christian Democratic movement, his father, André Fontaine, was a very influential journalist and historian, specialist on the Cold War. He worked from 1947 to 1991 for the well-known daily newspaper Le Monde, where he served in particular as editor-in-chief (1969-85) and director (1985-91).

Jean-Marc himself had a natural taste for intellectual debates, and very independent judgement. In most cases, his point of view was original, sometimes almost provocative.

This is already apparent in the choices he made when he was a student at École Polytechnique, from 1962 to 1965. Indeed, high-level teaching and research in mathematics were still somewhat undeveloped at École Polytechnique in the late fifties. However this began to change with the appointment of Laurent Schwartz as a professor in 1958.

Communicated by Notices Associate Editor William McCallum.

For permission to reprint this article, please contact:

reprint-permission@ams.org.

DOI: https://doi.org/10.1090/noti2118

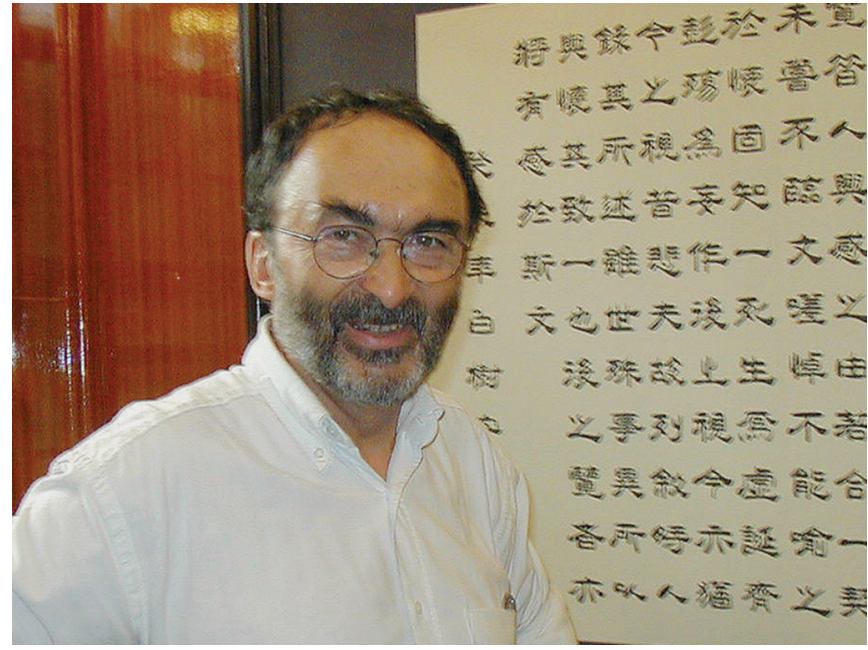

Figure 1. Jean-Marc Fontaine during a visit to China, standing next to a modern copy of the "Lantingji Xu".

In just a few years, Schwartz succeeded in creating an active mathematical research center, and became very influential among the students who wanted to specialize in mathematics. It is no surprise that many chose a thesis topic in analysis. However, Jean-Marc became more interested in number theory, and it is by attending the lectures of Charles Pisot that he found his path towards mathematical research.

Pisot was Jean-Marc's advisor for two years. In December 1967, Jean-Marc gave a lecture at the Delange-PisotPoitou seminar on ramification for local field extensions [1]. Jean-Pierre Serre was very positively impressed. He got in touch with Jean-Marc, and, as a thesis topic, he proposed his conjecture on the rationality of Artin representations of local fields. Jean-Marc proved the conjecture pretty rapidly and defended his thesis in 1972 [2]. Then, 
with the encouragement of Serre, he began to develop a program for a systematic study of $p$-adic representations of Galois groups of local fields, and this is where the story of Fontaine rings begins.

\section{Jean-Marc Fontaine}

\section{Nicholas M. Katz}

I first met Jean-Marc Fontaine and his family in the early 1970s. Our first children were born the same year (1974), and they remain friends to this day. In the following years we made several family visits to them in Grenoble, and had many dinners together when we were at IHES and they were in Paris.

Let me say a few words about some of the mathematics we owe to Jean-Marc. His work is described in detail in the articles of Berthelot and Scholze, but I want to give a "baby" version of one part, and a reminiscence of the impact it had. From the early 1970s, Jean-Marc was fascinated by Grothendieck's "mysterious functor." Recall the background of this.

Tate in 1966 had shown that a $p$-divisible group $\Gamma$ over the ring of integers, (e.g., $\mathbb{Z}_{p}$ ) of a local field (e.g., $\mathbb{Q}_{p}$ ) was determined by its Galois representation (the action of $\operatorname{Gal}\left(\overline{\mathbb{Q}_{p}} / \mathbb{Q}_{p}\right)$ on the Tate module formed from the $p$-power torsion points of $\Gamma\left(\overline{\mathbb{Q}_{p}}\right)$ ). On the other hand, Messing, carrying out an idea of Grothendieck, had shown that for $p>$ 2 , such a $\Gamma$ was determined by a filtration of the Dieudonné module of the special fibre $\Gamma_{\mathbb{F}_{p}}$ a $p$-divisible group over $\mathbb{F}_{p}$. The "mysterious functor" was to pass between the Galois representation and the filtered Dieudonné module. The mystery was how to do this without invoking $\Gamma$. When, for example, $\Gamma$ is the $p$-divisible group of an abelian variety $A / \mathbb{Q}_{p}$ with good reduction (i.e., an abelian scheme $\mathbb{A} / \mathbb{Z}_{p}$ ), then the Galois representation is the $p$-adic étale cohomology group $H_{\text {ét }}^{1}$ of $A_{\overline{\mathbb{Q}}_{p}}$, while the Dieudonné module is the de Rham cohomology group $H_{\mathrm{dR}}^{1}$ of $\mathbb{A} / \mathbb{Z}_{p}$. So more generally, the "mysterious functor" was to pass between the $p$-adic étale cohomology and the filtered de Rham cohomology of (eventually quite general) varieties over local fields of mixed characteristic $(0, p)$.

At the Journées de Géométrie Algébrique de Rennes in 1978, Jean-Marc astounded all of us by introducing his "Barsotti-Tate rings," which, he showed, realized the "mysterious functor" in the case of $p$-divisible groups. This not only solved an existing problem, but was a revolution in

Nicholas M. Katz is a professor of mathematics at Princeton University, Princeton, New Jersey. His email address is nmk@math.princeton.edu. how to think about such problems. Further development of his ideas led to more general rings of this type, which are some of the fundamentals of $p$-adic Hodge theory, but this was the starting point.

I refer to Illusie's piece for his keen insight into JeanMarc as a colleague and as a courageous human being. It was an honor to have known Jean-Marc. He will be sorely missed.

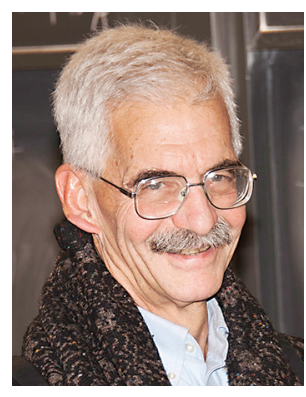

Nicholas M. Katz

\section{Remembering Jean-Marc}

\section{Luc Illusie}

I am not sure when I met Jean-Marc for the first time. It was probably at his 1972 thesis defense, which was directed by Serre, about the rationality of Artin representations. That summer we both attended the Antwerp summer school on modular forms, and two years later we were both at the AMS Summer Institute on Algebraic Geometry, in Arcata. I also attended his Cours Peccot at Collège de France in 1975. After his thesis, Jean-Marc took a position at Grenoble. In 1988, Jean-Marc moved to Orsay, where he was a professor until his retirement in 2009, when he became emeritus.

His Cours Peccot was about the classification of $p$ divisible groups over local fields. He was fascinated by questions of Grothendieck about $p$-divisible groups and the "mysterious functor" relating de Rham cohomology and $p$-adic cohomology of varieties over local fields. At that time, no one had the slightest idea of how to proceed, or even how to formulate precise conjectures. Jean-Marc

Luc Illusie is a retired professor of mathematics at Université Paris-Saclay, Orsay, France. His email address is 1uc. 117 usi e@wanadoo.fr.

This text is the translation by Nicholas M. Katz of a slightly edited version of the eulogy delivered by the author at Jean-Marc Fontaine's funeral on February 5, 2019, which appeared in the October 2019 issue of La Gazette des Mathématiciens, and is translated and published here by permission. The author is very grateful to Nick Katz for the translation of his piece, and thanks the editors of the Gazette for granting the Notices permission to include this translation. 


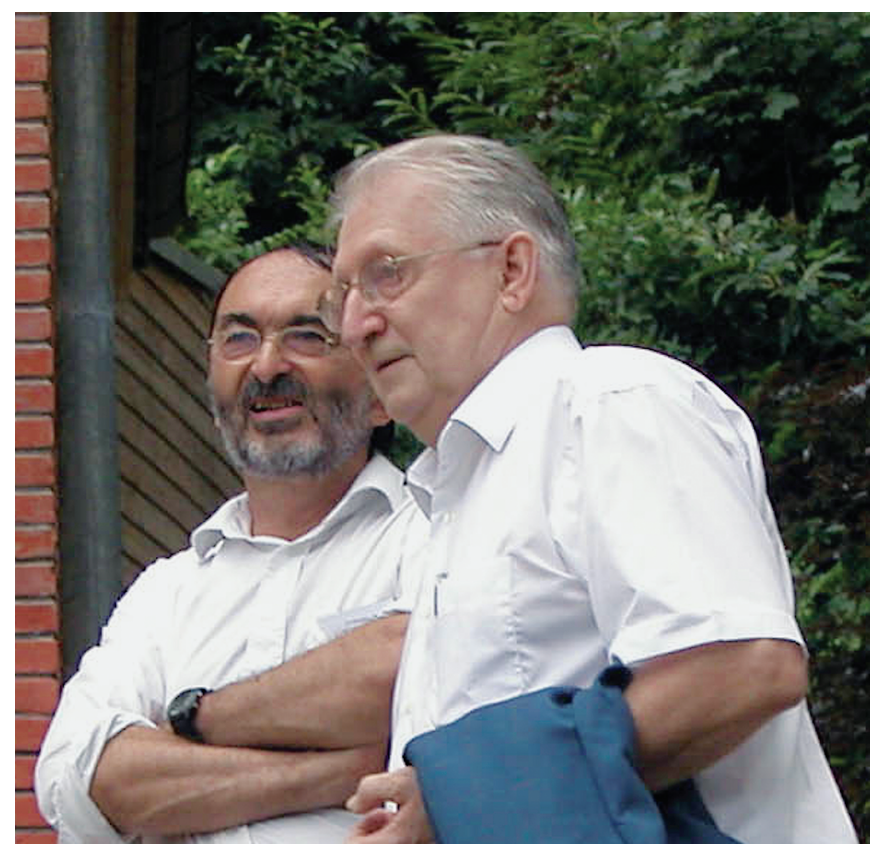

Figure 2. Jean-Marc Fontaine with Michel Raynaud (Conference in honor of L. Illusie, IHES, 2005).

devoted himself to this, and over the next years constructed an elaborate algebraic formalism in which the conjectures could be formulated, and which eventually led to their complete solution. His formalism, based on the construction of certain rings called "period rings" which now bear his name, was at first received with a combination of skepticism and fear. It became the pillar of $p$-adic Hodge theory. His rings play a central role in present-day arithmetic geometry. Over the past twenty years, they have had many spectacular applications, featuring in the work of TaylorWiles on Fermat, and of Khare-Wintenberger on Serre's modularity conjecture.

Throughout my career, I have been closely involved with Jean-Marc, not only at Orsay but also as we traveled together all around the (mathematical) world: Europe, the US, India, China, Japan. Let me say a few words about his arrival at Orsay in 1988. It brought us a breath of fresh air, a renewed sense of energy. Together with Raynaud, Wintenberger $^{1}$ (who had himself just arrived at Orsay), PerrinRiou, Kato (who was visiting Orsay), and Mazur, who was visiting IHES, Jean-Marc organized a seminar at IHES on "p-adic periods." There was great enthusiasm about the "semistable conjecture" ${ }^{2} C_{s t}$ of Jean-Marc and Jannsen. The geometric formulation of $C_{s t}$, following work of Hyodo and Kato, led to new subjects of research, in particular what is now called logarithmic geometry. ${ }^{3}$ The book

\footnotetext{
${ }^{1}$ Sadly deceased on January 23, 2019.

${ }^{2}$ It was fully proven some twelve years later by Tsuji.

${ }^{3}$ In fact, logarithmic geometry was born in a conversation Jean-Marc and I had
}

Périodes p-adiques, Astérisque 223, which grew out of the seminar, remains today a basic reference for $p$-adic Hodge theory.

The 1990s were also rich in new developments: work of Jean-Marc and Perrin-Riou on Bloch-Kato, formulation of the Fontaine-Mazur conjecture, a " $p$-adic semester" at IHP in spring 1997 co-organized with Berthelot, Kato, Rapoport, and me and devoted in particular to work of Tsuji and Faltings on $p$-adic comparison theorems.

Let me now turn to Jean-Marc's interaction with China. After going to Beijing to give an invited talk at the 2002 ICM, Jean-Marc thought it was the right time to start a cooperative program with China in arithmetic geometry. This was realized by a chaired professorship of arithmetic geometry at Tsinghua University which ran from 2003 to 2006, of which Jean-Marc was the first. He convinced several of his Orsay colleagues (including me) to go teach at Tsinghua, to give a solid foundation to students who were both talented and anxious to learn. Each year, some of the best were chosen to come to Orsay or ENS to write a thesis. Many then returned to China to hold permanent academic positions. They became the "dorsal fin" of arithmetic geometry in China. Jean-Marc was the guiding spirit of this cooperation, to which he devoted all his energy until he fell ill in 2016. In an article published this past January 30 by the Chinese Mathematical Society, Yi Ouyang (who gave us invaluable assistance in Beijing, and who was a coauthor with Jean-Marc of an introductory book on $p$ adic Hodge theory) wrote a moving tribute ${ }^{4}$ to Jean-Marc. Miaofen Chen, who had been our student in Tsinghua in 2004, and is now a professor at ECNU in Shanghai, made a video, together with former classmates, in Jean-Marc's honor in December of 2018.

Jean-Marc was extremely generous. He loved to convey his ideas and his vision of mathematics. This was of great benefit to his students, especially Wintenberger, Colmez, and Breuil, each of whom went on to contribute enormously to both his work and its influence.

Very much like Michel Raynaud, whom we lost in March of 2018, Jean-Marc did not care for honors, and never spoke of those he had received (invited speaker at the 1983 ICM in Warsaw and the 2002 ICM in Beijing, Prix Petit d'Ormoy, Carrière et Thébaut in 1984, Gay-Lussac Humboldt Prize in 2002, election to the Académie des Sciences in 2002).

Let me end on a personal note. Jean-Marc loved to argue about all sorts of things. At the Orsay dining hall, or around the coffee machine, we would have long

in July 1988, on the train taking us to Oberwolfach.
${ }^{4}$ This tribute is essentially reproduced in Ouyang's piece "The China legacy
of Jean-Marc Fontaine" in the October 2019 issue of La Gazette des Mathématiciens. 
discussions. I could barely state an opinion before JeanMarc objected; often he was right. But in November 2017, after his major surgery, I noticed a change. We were both in Padova, each giving a course. He knew his way around the back streets; I did not. On rainy nights we would go by means of narrow, winding, poorly lit streets to a good restaurant he knew. When we got there he wouldn't eat much but we talked for a long time. And we agreed on everything. It was a bad omen.

Right up to the end, Jean-Marc fought valiantly against the disease which was destroying his body. In March of 2016 I was in Taipei, and suggested Jean-Marc to Chia $\mathrm{Fu} \mathrm{Yu}$, who then invited him to come give a course. He accepted, but had to postpone coming several times, because of his operation and its ensuing complications. He finally went in October of 2018, despite his cancer having returned with a vengeance. With incredible stoicism, he gave three two-hour lectures, before having to be rushed home.

In our last conversations, we talked as though everything were normal-about math, about current events, about music, about Schoenberg's La nuit transfigurée, a favorite of his. Some mathematical things he cared deeply about were the re-edition, with errata and addenda, of the book Périodes p-adiques, Astérisque 223; the publication of his last article, ${ }^{5}$ on almost $C_{p}$-representations; the publication of his book with Yi Ouyang; and the publication of his work with Fargues on the "fundamental curve" in $p$ adic Hodge theory. Of these, only the last, Asterisque 406, was done in time for Jean-Marc to hold in his hands.

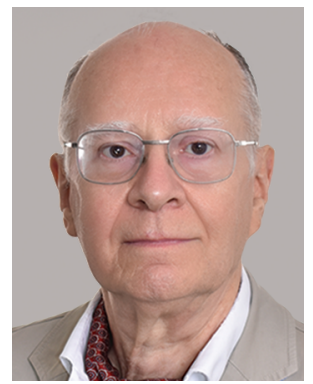

Luc Illusie

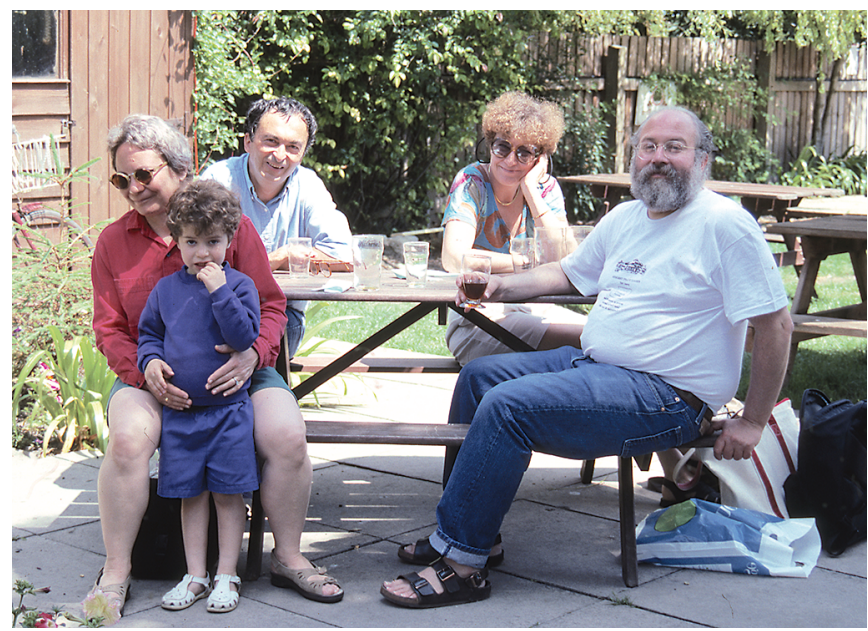

Figure 3. Jean-Marc Fontaine in Cambridge (UK), with the Messing and Berthelot families, after Wiles's lectures on Fermat (June 28, 1993).

\section{Souvenirs de Jean-Marc}

\section{William Messing}

I first encountered Jean-Marc on Monday, June 7, 1971 when he was sitting with Serre during the pot before Serre's Séminaire Bourbaki talk. I next encountered him on Monday, June 5, 1972, again sitting with Serre, before Serre's Séminaire Bourbaki talk. I do not recall speaking to him on either of these occasions. That summer, we both attended the Antwerp Modular Forms of One Variable for three weeks and we did discuss $p$-divisible groups a couple of times.

It was during the AMS Summer Institute in Algebraic Geometry, held in Arcata in August 1974, that I really got to know Jean-Marc. He was there with his first wife, Laurence, and their infant daughter, Isabelle, and I discussed with him our common interests in $p$-divisible groups, $p$ adic Galois representations, and Grothendieck's problem of the "mysterious functor." He attended my two lectures and I was very impressed by his August 15 lecture, given in the Serre-Tate seminar, Arithmetic, Formal Groups and their Division Points.

I visited Berthelot and Breen in Rennes during the period September-December 1976. In November 1976 I flew from Rennes to Lyon, where Jean-Marc picked me up at the airport and drove to his Grenoble apartment where I spent a week staying with him, Laurence, and their three daughters. The twins, Camille and Caroline, were infants

William Messing is a professor of mathematics at the School of Mathematics, University of Minnesota, Minneapolis, Minnesota. His email address is messing@math.umn. edu.
${ }^{5}$ This article has now appeared: Jean-Marc Fontaine, Almost $\mathbf{C}_{p}$ Galois representations and vector bundles, Tunis. J. Math. 2 (2020), no. 3, 667-732. MR404128c. 
at the time. It was a terrific visit, both because of our mathematical interaction and my getting to know both him and Laurence as wonderful hosts and friends. JeanMarc was then a believing Catholic. I was surprised, as the French mathematicians whom I had known previously were strongly anticlerical. He told me a wonderful story about how in the past he had traveled with four priests to Normandy, where they enjoyed a large sumptuous luncheon on a Friday featuring Marmite Dieppoise, agneau de pré-salé, camembert, and several trous normands. I asked Jean-Marc about the priests eating meat on a Friday, and he told me that he had asked them the same question and their response was "The peasants refrain from eating meat; the priests do not." I was both amused and amazed with this story, as he was in recounting it.

On Thursday and Friday, July 6 and 7, 1978, Jean-Marc spoke at the Rennes conference, Journées de Géométrie Algébrique de Rennes. Jean-Marc announced and outlined his solution to the mysterious functor problem for abelian varieties and $p$-divisible groups. To put it mildly, this was a striking result and was, hands down, the highlight of the conference. I spent the summer and autumn first at the IHES and then in Rennes. Jean-Marc visited Rennes and stayed at chez Berthelot for a week. The three of us talked at length. I again visited Jean-Marc in Grenoble, staying again with him and Laurence.

The Fontaine family spent the month of January 1980 visiting my wife, Rita, and me at UC Irvine. We had rented a small bungalow for them on Balboa Island. Jean-Marc and I began seriously working together during that month. Let $k$ be a perfect field of characteristic $p>0$, let $W_{n}$ be the Witt vectors of length $n$ of $k$, and let $S_{n}=\operatorname{Spec}\left(W_{n}\right)$. He told me that, with covering morphisms being flat, locally complete intersections the functor assigning to a scheme $X / k, H_{c r y s}^{0}\left(X / S_{n}\right)$ is a sheaf. I told him that this topology had previously been considered by Mazur, who named it the syntomic topology, and I gave Jean-Marc a copy of Mazur's unpublished notes on the syntomic topology. We named this sheaf $\mathcal{O}_{n}^{\text {crys }}$. The next day, I gave Jean-Marc a proof that if $X_{S Y N}$ denotes the big syntomic site of the $k$ scheme $X$, then we have a canonical isomorphism, compatible with the action of Frobenius, between $H_{c r y s}^{*}\left(X / S_{n}\right)$ and $H^{*}\left(X_{S Y N}, \mathcal{O}_{n}^{\text {crys }}\right)$. He was delighted and our work took off.

The Messing family spent the period June-December 1980 abroad. Jean-Marc and I corresponded that summer and, in particular, on August 19, I wrote the letter permitting the formulation of the $C_{c r y s}$ conjecture in JeanMarc's Annals of Mathematics paper. In September, my nine-year-old son, Charles, and I went to Rennes, where he entered the école primaire near the Berthelot house. Each afternoon, after school, he would play with the Berthelot children, Katell and Erwan, until I picked him up and we drove to our small apartment in Zup-Sud Rennes. JeanMarc visited Rennes and again stayed with the Berthelots. We continued to work together.

During the Fall Semester 1983, I visited Jean-Marc in Grenoble and stayed at the Institut Fourier tower where there were a small number of apartments. Jean-Marc and I discussed Dieudonné gauges. Jean-Marc was an Ordway Visitor at the Minnesota Mathematics Department and stayed at the Messing house November 12-December 11, 1984. We continued to work on $p$-adic comparison theorems. He gave a series of lectures on Wintenberger's theorem showing when $K$ is a characteristic zero, absolutely unramified local field with perfect residue field of characteristic $p>0$ and $X / K$ is proper and smooth and has good reduction, the Hodge filtration on $H_{d R}^{*}(X / K)$ is canonically split.

I saw Jean-Marc next at the conference for Tate's sixtieth birthday at Harvard, in April 1985. Jean-Marc and I worked intently at a blackboard for three days and then Jean-Marc lectured on the comparison theorem we had proven. The AMS conference Current Trends in Arithmetic Algebraic Geometry was held in Arcata, August 1824,1985 . I gave four lectures on our joint work and our paper was published in the proceedings of that conference. Immediately after the conference, Jean-Marc came to our house in Saint Paul, where he stayed a week and gave a beautiful lecture on the Frey curve and Serre's strategy for proving Fermat's Last Theorem. Shortly after, I returned to Grenoble, where I again spent the Fall Semester. In addition to Jean-Marc's presence, Kato was also a visitor and this was when I first met him. Kato learned and immediately understood what Jean-Marc and I had done and saw how to generalize some of our results. It was thrilling for both Jean-Marc and me to see and learn from a young master.

Jean-Marc and I exchanged emails in November 2018. I wrote to him on November 11 and he responded on November 22. Here are those letters:

Cher Jean-Marc,

Please permit me to write in English, as I want to be as precise as possible. Luc wrote and told me of your relapse and the lack of hope for your recovery. I have recalled and reflected on the more than forty-four years we have known each other, my visits to you in Grenoble, your visits to me in both Irvine and Minnesota. When I last saw you at the Gabber conference in June, you seemed to be in good spirits and I was very happy that you were a cancer survivor. Thus, I was shocked to learn of the recurrence. I remember vividly the hospitality you and Laurence extended to me in Grenoble and getting to know both of you and your daughters. Later getting to know Hélène. I remember how much I learned from you, your incredible drive and 
work ethic and mathematical vision which reshaped the p-adic landscape. I recall my interest in your Catholic faith, as most mathematicians I knew were noncroyant. I remember too being quite surprised when you told me, that you had stopped going to Mass each week. Please let me know if there is anything that Rita and I might do to aid and bring you solace.

Amitiés,

Bill

\section{Dear Bill,}

Sorry for being so slow to answer but I was too tired to write anything reasonable. Thank you heartily for your message which touched me deeply. Needless to say that, despite my bad temper, it has been a pleasure to work with you. I know very well that our joint work on p-adic comparison theorems has been the hardest thing I ever contributed to and that, without you, I would have had no chance to get into this kind of thing. That we did not succeed to continue to work together is entirely my fault and I regret it deeply. I also have a lot of nice souvenirs of when we were together, in particular of your warm hospitality with Rita in Minneapolis. Everything concerning my catholic faith seems to me now as if it never existed. I am ready to die (even if I would love to wait 20 more years!). I am neither interested or frightened by what is going to happen after. I can not say the same about what is going to happen before. I wish you, Rita and your family a long, nice and peaceful life.

With all my affection,

Jean-Marc

Adieu Jean-Marc.

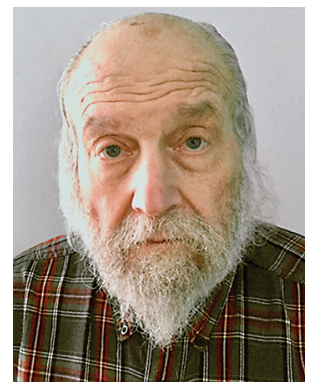

William Messing

\section{A Quest for Rings}

\section{Pierre Berthelot}

At the heart of Fontaine's program for the study of $p$-adic representations of Galois groups of local fields, Fontaine's rings have often be viewed as mysterious and highly impressive objects. Yet, their construction has been the result of a very coherent strategy, initiated by Fontaine in the early seventies, and regularly enriched with new viewpoints and key results. In this survey, I would like to put into perspective the main steps of these developments, from the early contributions of Tate and Serre to the complete solution of Fontaine's conjectures some thirty years later.

In the whole story, $p$ denotes a fixed prime number, and $K$ a local field ${ }^{6}$ of characteristic 0 and residue characteristic $p$ ( $K$ is also called a $p$-adic field). Let $\bar{K}$ be an algebraic closure of $K, C$ its completion, $O_{K}, O_{\bar{K}}, O_{C}$ the rings of integers in $K, \bar{K}, C$, and $k$ the residue field of $O_{K}$. When needed, the result of a base change will be denoted by adding the new base as a subscript.

The objects of interest here are the $p$-adic representations $^{7}$ of the absolute Galois group $G=\operatorname{Gal}(\bar{K} / K)$. Let me first recall a few important results already known in the late sixties.

\section{Hodge-Tate Representations}

A simple example of a $p$-adic representation of $G:=$ $\operatorname{Gal}(\bar{K} / K)$ is given by the natural action of $G$ on the roots of unity in $\bar{K}$ : use the transition maps $\mu_{p^{n+1}}(\bar{K}) \rightarrow \mu_{p^{n}}(\bar{K})$ given by elevation to the $p$ th power to define

$$
T_{p}(\mu):=\underbrace{\lim }_{n} \mu_{p^{n}}(\bar{K}), \quad V_{p}(\mu):=\mathbb{Q}_{p} \otimes_{\mathbb{Z}_{p}} T_{p}(\mu)
$$

Then $V_{p}(\mu)$ is a 1-dimensional $\mathbb{Q}_{p}$-representation of $G$, defined by the ( $p$-adic) cyclotomic character $\chi$ of $G$. For any $i \in$ $\mathbb{Z}$, we denote by $\mathbb{Q}_{p}(i)$ the $\mathbb{Q}_{p}$-representation of dimension 1 given by multiplication by $\chi^{i}$. If $E$ is a $\mathbb{Q}_{p}$-representation of $G$, we denote by $E(i)$ the $\mathbb{Q}_{p}$-representation $E \otimes_{\mathbb{Q}_{p}} \mathbb{Q}_{p}(i)$, and by $E\{i\}$ the subspace $\left\{x \in E \mid \forall g \in G, g x=\chi(g)^{i} x\right\}$.

Pierre Berthelot is a retired professor of mathematics at Université de Rennes 1 , Rennes, France. His email address is pierre.berthelot@univ-rennes1 . fr.

This text is partly based on an unpublished report due to Luc Illusie. The author thanks him heartily for letting him use that document.

${ }^{6}$ i.e., a complete discrete valuation field with perfect residue field.

${ }^{7}$ i.e., the finite-dimensional $\mathbb{Q}_{p}$-vector spaces endowed with a continuous linear action of $G$. We will often write simply " $\mathbb{Q}_{p}$-representation." 
Let $V$ be a $\mathbb{Q}_{p}$-representation of $G$. Then the $C$-vector space $C \otimes_{\mathbb{Q}_{p}} V$ is endowed with a semilinear action of $G$, and $\left(C \otimes_{\mathbb{Q}_{p}} V\right)\{i\}$ is a $K$-subspace of $C \otimes_{\mathbb{Q}_{p}} V$. A fundamental result of Tate on the Galois cohomology of $C$ implies that the $G$-equivariant map

$$
\varepsilon: \bigoplus_{i} C \otimes_{K}\left(\left(C \otimes_{\mathbb{Q}_{p}} V\right)\{i\}\right) \rightarrow C \otimes_{\mathbb{Q}_{p}} V
$$

is injective (see [Serre, MR0242839] and [Tate, MR0231827]). One says that $V$ is a Hodge-Tate representation if $\varepsilon$ is an isomorphism. The integers $i$ for which $(C \otimes V)\{i\} \neq 0$ are then called the Hodge-Tate weights of $V$.

Let $H=\bigcup_{n} H[n]$ be a $p$-divisible group ${ }^{8}$ of height $h$ over $K, H[n]$ denoting the kernel of multiplication by $p^{n}$ in $H$. The above construction can be repeated using the subgroups $H[n]$ in place of the $\mu_{p^{n}}$ 's. This gives the Tate module $T_{p}(H)$ and the associated $p$-adic representation $V_{p}(H)$ of $G$, of dimension $h$.

A natural question is then: is $V(H)$ a Hodge-Tate representation? When $H$ has good reduction over $O_{K}$, i.e., is the generic fiber of a $p$-divisible group $\mathcal{H}$ on $O_{K}$, Tate gave a positive answer by constructing a $G$-equivariant isomorphism

$$
t_{\mathcal{H}^{\prime}}(C) \oplus\left[t_{\mathcal{H}}^{*}(C) \otimes V_{p}(\mu)^{\vee}\right] \stackrel{\sim}{\rightarrow} V(H)^{\vee},
$$

where $\vee$ denotes the $C$-valued dual, $\mathcal{H}^{\prime}$ is the Cartier dual of $\mathcal{H}$, and $t$ (resp., $t^{*}$ ) denotes the tangent (resp., cotangent) space [MR0231827]. Using the semistable reduction theorem [SGA7], Raynaud extended Tate's proof to the case of the $p$-divisible group $H=A[\infty]$ defined by an arbitrary abelian variety $A$. In 1982, Fontaine gave another proof for p-divisible groups, using a completely different method based on the study of the modules of absolute 1-forms of $O_{K}$ and $O_{\bar{K}}[6]$.

When $H=A[\infty], V(H)^{\vee}$ can classically be identified with the first étale cohomology group $H_{\text {èt }}^{1}\left(A_{\bar{K}}, \mathbb{Q}_{p}\right)$. Tate's isomorphism can then be written

$$
\begin{aligned}
\left(H^{1}\left(A, \mathcal{O}_{A}\right) \bigotimes_{K} C\right) \oplus\left(H^{0}\left(A, \Omega_{A}^{1}\right)\right. & \left.\otimes_{K} C(-1)\right) \\
& \stackrel{\sim}{\rightarrow} C \otimes_{\mathbb{Q}_{p}} H_{\text {èt }}^{1}\left(A_{\bar{K}}, \mathbb{Q}_{p}\right) .
\end{aligned}
$$

Tate asked whether a similar decomposition might actually exist for any proper and smooth $K$-scheme, thus giving the first formulation of the Hodge-Tate decomposition conjecture:

\footnotetext{
$8_{i . e .,}$ a direct system of finite locally free commutative group schemes $H[n]$ over $S$, of rank $p^{n h}$, such that the transition maps identify $H[n]$ with the kernel of multiplication by $p^{n}$ on $H[n+1]$. Following Grothendieck, $p$-divisible groups are also called Barsotti-Tate groups.
}

Conjecture/Theorem $\mathbf{1}\left(\mathbf{C}_{\mathbf{H T}}\right)$. Let $X$ be a proper and smooth scheme over $K$. For any $n \in \mathbf{N}$, there exists a canonical $G$ equivariant isomorphism

$$
\bigoplus_{i+j=n} H^{j}\left(X, \Omega_{X / K}^{i}\right) \otimes_{K} C(-i) \stackrel{\sim}{\rightarrow} C \otimes_{\mathbb{Q}_{p}} H_{\mathrm{ét}}^{n}\left(X_{\bar{K}}, \mathbb{Q}_{p}\right) .
$$

We'll see later how this conjecture has been included in the broader context of Fontaine's conjectures. By analogy with the Hodge decomposition for the cohomology of complex varieties, the subject became known as $p$-adic Hodge theory.

\section{The "Mysterious Functor"}

Another major input for the development of $p$-adic Hodge theory came from Grothendieck's work on Dieudonné theory for $p$-divisible groups (cf. his lecture at ICM70 in Nice [MR0578496] and his 1970 Montreal course [MR0417192]).

Dieudonné theory was initiated by a series of articles written by Dieudonné in the fifties. Their goal was to classify formal Lie groups over a perfect field $k$ of characteristic $p$ by some kind of (semi)linear data analogous to the Lie algebra in characteristic 0 . It has been developed by many authors, in several flavors, covariant or contravariant, each with its own hypotheses.

Over $k$, one of the most commonly used constructions is due to Manin (see Manin [MR0157972] or Demazure [MR0344261]). Let $W=W(k)=\lim _{n} W_{n}(k)$ be the ring of Witt vectors with coefficients in $k$ and let $\sigma$ be its Frobenius automorphism. For a finite unipotent $p$-group scheme $\Gamma$ over $\operatorname{Spec}(k)$, Manin defines the Dieudonné module $M(\Gamma)$ by $M(\Gamma):=\operatorname{Hom}(\Gamma, \mathbb{W})$, where $\mathbb{W}$ is the ind-group scheme representing the "ring of Witt vectors functor." Then $M(\Gamma)$ is a finitely generated torsion $W$-module, endowed with a pair of endomorphisms $F, V$, respectively, $\sigma$ and $\sigma^{-1}$. semilinear, such that $F V=V F=p$ and $V$ is nilpotent. The functor $\Gamma \mapsto(M(\Gamma), F, V)$ is an equivalence of categories. By an inverse limit argument, it yields an equivalence between unipotent $p$-divisible groups and free finitely generated $W$-modules endowed with $F, V$ as before, $V$ being now topologically nilpotent. Finally, the theory can be extended to all finite or $p$-divisible $k$-groups using the canonical decomposition of a $p$-group scheme on a perfect field and Cartier duality.

Grothendieck's interest in Dieudonné theory was motivated by the development of crystalline cohomology in a relative situation, and by the possibility of using deformation theory to go from characteristic $p$ to characteristic 0 . His idea was that over a nonperfect base $S$, one should replace the Dieudonné module of a $p$-divisible group $H$ by a Dieudonné crystal $\mathbb{D}(H)$. This would provide a locally 
free finitely generated $\mathcal{O}_{S^{\prime}}$-module $\mathbb{D}(H)_{S^{\prime}}$ for any nilimmersion $S \hookrightarrow S^{\prime}$ defined by an ideal endowed with a suitable divided power structure, ${ }^{9}$ together with base change isomorphisms when $S^{\prime}$ varies. For $S=\operatorname{Spec} k$, the datum of a crystal on $S$ is equivalent to that of a $p$-adically complete and separated $W$-module, and $\mathbb{D}(H)$ should give back the usual Dieudonné module.

Grothendieck proposed two constructions of the crystal $\mathbb{D}(H)$, which yield in addition a locally split monomorphism $\omega_{H} \hookrightarrow \mathbb{D}(H)_{S}$, where $\omega_{H}$ is the module of invariant differential forms on $H$. His program was completed by Messing |MR0347836 (see also [MazurMessing, MR0374150|), who proved in particular that if the divided powers satisfy some nilpotency condition, the datum of a $p$-divisible group $H^{\prime}$ on $S^{\prime}$ lifting $H$ is equivalent to the datum of a locally split submodule $\mathcal{L} \subset \mathbb{D}(H)_{S^{\prime}}$ lifting $\omega_{H} \hookrightarrow \mathbb{D}(H)_{S}$.

Assume again that $K$ is a $p$-adic field with residue field $k$. Let $K_{0}$ be the fraction field of $W$, which is in a natural way a subfield of $K$. Using Messing's theorem, one gets that, up to isogeny, a $p$-divisible group $H$ over $O_{K}$ is determined by the data of a $K_{0}$-vector space $E$ endowed with a $\sigma$-semilinear automorphism $F$, and of a $K$-subspace $L \subset K \otimes_{K_{0}} E$. It follows that the $p$-adic representation $V\left(H_{K}\right)$ is determined uniquely by the triple $(E, F, L)$. But the converse is also true, as Tate proved in [MR0231827] that the functor $H \mapsto H_{K}$ is fully faithful.

Then Grothendieck emphasized the following striking fact. Using the theory of $p$-divisible groups on $O_{K}$ as an intermediate, we obtain a correspondence between two very different kinds of data. However, both are described in purely algebraic terms, without ever using the notion of a $p$-divisible group, nor any other geometric construction. This led him to ask in his ICM70 talk [MR0578496 the following questions:

(1) Can one give a direct construction of the $p$-adic representation $V\left(H_{K}\right)$ in terms of the data $(E, F, L)$, without using $p$-divisible groups? Conversely, is there a direct construction recovering the triple $(E, F, L)$ from $V\left(H_{K}\right)$ ?

(2) Is there a higher dimension analogue of this correspondence relating, for a proper and smooth $O_{K}$-scheme $X$, the $p$-adic representations $H_{\mathrm{et}}^{*}\left(X_{\bar{K}}, \mathbb{Q}_{p}\right)$ with the crystalline cohomology spaces $H_{\text {cris }}^{*}\left(X_{k} / W\right) \otimes_{W} K_{0}$ endowed with their Frobenius action and a Hodge-type filtration?

\footnotetext{
${ }^{9} A$ divided power structure, or PD-structure, on an ideal $J$ is a family of operators on $J$, often denoted by $x \mapsto x^{[n]}$, which behave "as the operators $x \mapsto$ $x^{n} / n$ ! in characteristic 0" (see [MR0384804 or MR0491705]). Endowed with this extra structure, $J$ is called a PD-ideal. For example, for any $\mathbb{Z}_{p}$-algebra $R$, the ideal $p R$ has a canonical PD-structure given by $(p x)^{[n]}=\left(p^{n} / n !\right) x^{n}$ for all $n \geq 1$ and all $x \in R$. We always require that a PD-structure on $J$ induce the canonical PD-structure on $J \cap p R$.
}

In 1970, these questions seemed to be completely inaccessible. Following Grothendieck, they were referred to as the problem of the mysterious functor.

\section{Dieudonné Theory Revisited}

During a lecture at IHES ${ }^{10}$ in 2009, Fontaine pointed out that he met Grothendieck only once: in 1972, during the Antwerp Summer School on Modular Functions. As is well known, ${ }^{11}$ there could have been better circumstances for an introduction to the problem of the mysterious functor. Fortunately, Serre took over: he shared with Fontaine his interest in these questions, and he convinced him to work on their solution.

Fontaine's strategy unfolded progressively. Its first step was to revisit completely the previous results about the classification of finite $p$-groups and $p$-divisible groups over $k$ and $O_{K}$. More specifically, Fontaine's goal was to make as explicit as possible the construction of a quasi-inverse functor retrieving the initial $p$-divisible group from the classifying data.

Over $k$, Manin's construction of the Dieudonné module has an unpleasant feature: in order to define the Dieudonné module of an arbitrary $p$-divisible group $H$, one must decompose $H$ as the product of its unipotent and multiplicative components and give an ad hoc definition for each of the two components. Fontaine looked for an alternate definition that would unify the treatment of the two cases. His solution was to modify a construction of Barsotti and to use the sheaf of Witt covectors. If $R$ is a $k$-algebra, define

$$
C W(R)=\left\{\begin{array}{r}
\left(\ldots, a_{-i}, \ldots, a_{-1}, a_{0}\right) \mid a_{-i} \in R, \\
\exists r>0 \text { such that } \\
\text { the ideal }\left(a_{-i}\right)_{i \geq r} \text { is nilpotent }
\end{array}\right\} .
$$

Let $D_{k}$ be the Dieudonné ring, generated by $W$ and by elements $F, V$, with relations $F V=V F=p, F a=$ $\sigma(a) F, V \sigma(a)=a V$ for all $a \in W$. Then $C W(R)$ has a functorial structure of left- $D_{k}$-module.

Let $\mathcal{A}$ be the category of finite $k$-algebras. Fontaine defines the Dieudonné module of a finite $p$-group scheme $H$ as being the left $D_{k}$-module $\mathcal{D}(H)=\operatorname{Hom}(H, C W)$, where Hom is the set of morphisms of group functors on $\mathcal{A}$. If $M$ is a $D_{k}$-module of finite length as a $W$-module, he also defines a functor $\mathcal{G}(M)$ on $\mathcal{A}$ by setting $\mathcal{G}(M)(R)=$ $\operatorname{Hom}_{D_{k}}(M, C W(R))$. Then he proves that the functors $H \mapsto$ $\mathcal{D}(H)$ and $M \mapsto \mathcal{G}(M)$ are left adjoint functors, providing an antiequivalence between the category of finite $p$-groups over $k$ and the category of $D_{k}$-modules of finite length over $W$. Taking into account appropriate topologies allows him to obtain similar results for $p$-divisible groups.

\footnotetext{
${ }^{10}$ Available at https: //www. dai 7 ymotion.com/video/x8jeru.

${ }^{11}$ See for example [Jackson, MR2104915 p. 1201].
} 
At this point, Fontaine has recovered the main results of classical Dieudonné theory over $k$, with an explicit description of a quasi-inverse functor $\mathcal{G}$. Then he proceeds with the classification of $p$-divisible groups over $O_{K}$, first up to isomorphism in the unramified case (for $p \neq 2$ ), next up to isogeny in the general case. We cannot give the details of his constructions here, but we would like to emphasize a feature that will become the core of his method for classifying $p$-adic representations: in order to define a pair of functors relating two different categories of algebraic structures, he constructs an object endowed with both structures in a compatible way, and uses bifunctors such as Hom or $\otimes$ to get functors in both directions. In the relation between finite $p$-group schemes over $k$ and Dieudonne modules, this role is played by the family of $\left(\mathbb{Z}_{p}, D_{k}\right)$-bimodules $C W(R)$. To give another example, we now sketch briefly the correspondence between isogeny classes of $p$-divisible groups over $O_{K}$ and triples $(E, F, L)$ as considered by Grothendieck.

For any discrete ring $A$, let $B W(A)$ be the additive group of Witt bivectors ${ }^{12}$ with coefficients in $A$; the functor $B W$ can be extended in a natural way to complete linearly topologized rings. When $A$ is a $k$-algebra, $B W(A)$ has a natural structure of a $D_{k}$-module. Denote by $\operatorname{Res}\left(O_{C}\right)$ the set of families $x=\left(x^{(n)}\right)_{n \in \mathbb{Z}}$ of elements of $O_{C}$ such that $\left(x^{(n+1)}\right)^{p}=x^{(n)}$ for all $n$. One can endow $\operatorname{Res}\left(O_{C}\right)$ with a structure of $k$-algebra, complete for a linear topology. Fontaine defines a $K_{0}$-linear homomorphism $\theta_{0}$ : $B W\left(\operatorname{Res}\left(O_{C}\right)\right) \rightarrow C$ by $\theta\left(\left(x_{n}\right)_{n \in \mathbb{Z}}\right)=\sum_{n \in \mathbb{Z}} p^{n} x_{n}^{(n)}$, and extends it by linearity as $\theta: K \otimes_{K_{0}} B W\left(\operatorname{Res}\left(O_{C}\right)\right) \rightarrow C$. Then he endows $K \otimes_{K_{0}} B W\left(\operatorname{Res}\left(O_{C}\right)\right)$ with the length 1 filtration defined by $\operatorname{Ker}(\theta)$, and constructs a $G$-equivariant isomorphism [3, V, Theorem 1]

$$
V(H) \stackrel{\sim}{\rightarrow}\left\{\begin{array}{r}
u \in \operatorname{Hom}_{K_{0}[F, V]}\left(E, B W\left(\operatorname{Res}\left(O_{C}\right)\right)\right) \\
\text { such that } u_{K}(L) \subset \operatorname{Ker}(\theta)
\end{array}\right\} .
$$

With these results, Fontaine answers Grothendieck's questions about the "mysterious functor" for $p$-divisible groups. They were the topic of his 1975 Cours Peccot at the Collège de France, and were published in 1977 as an Astérisque volume [3]. However, the method used for $p$ divisible groups could not suffice to construct the "mysterious functor" for varieties of any dimension, because it only gives representations with Hodge-Tate weights in $\{0,1\}$. So new ideas were again needed. ...

\section{Fontaine Rings I: $\mathbf{B}_{\mathrm{HT}}$}

In his quest for a correspondence relating the $p$-adic étale and de Rham/crystalline cohomologies of algebraic varieties over $K$, Fontaine had a guideline: these cohomologies

\footnotetext{
$12_{i . e .,}$ families $\left(x_{n}\right)_{n \in \mathbb{Z}}$ satisfying the condition used above to define $C W(A)$.
}

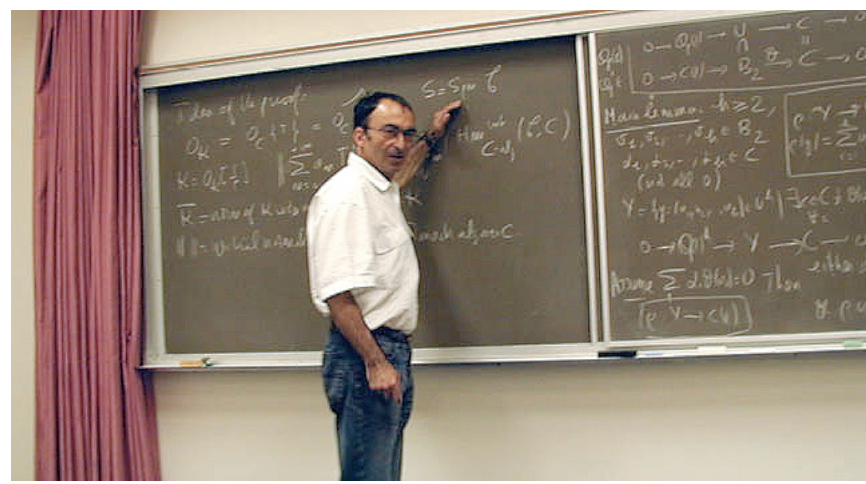

Figure 4. Jean-Marc Fontaine giving a lecture.

share some features, such as the Künneth formula or Poincare duality, and one could hope that the "mysterious functor" would respect these structures. More precisely, he wanted the functor to be an equivalence between two Tannakian categories, ${ }^{13}$ and to preserve the multiplicative structures on both. He concluded that the "bistructured objects" such as $C W(R)$ or $B W\left(\operatorname{Res}\left(O_{C}\right)\right)$ used in the construction of the "mysterious functor" for $p$-divisible groups should be replaced by similar objects having a ring structure.

With his lecture at the 1978 Rennes conference [5], Fontaine began to develop a very impressive program, which is still at the heart of $p$-adic Hodge theory nowadays. Let $\operatorname{Rep}(G)$ be the category of finite-dimensional continuous $\mathbb{Q}_{p}$-representations of $G$. Fontaine's strategy consists of describing various Tannakian subcategories of $\operatorname{Rep}(G)$ by means of correspondences with other tensor categories having as objects $K$-vector spaces endowed with some extra structures (such as a graduation, a filtration, etc.). Let $\mathcal{C}$ be such a tensor category. In each case, the correspondence is to be defined by an object of $\mathcal{C}$ endowed with a commutative $K$-algebra structure and a compatible $G$-action. For such algebras, which he calls $\left(\mathbb{Q}_{p}, G\right)$-rings, Fontaine uses the generic notation $B$ (as a tribute to Barsotti), generally enriched by some relevant abbreviation.

The algebra $B$ defines a functor $\mathcal{D}_{B}: \operatorname{Rep}(G) \rightarrow \mathcal{C}$ by setting, for any representation $V \in \operatorname{Rep}(G)$,

$$
\mathcal{D}_{B}(V)=\left(B \otimes_{\mathbb{Q}_{p}} V\right)^{G} .
$$

Let $F$ denote the $K$-algebra $B^{G}$. By adjunction, one gets a canonical $B$-linear map

$$
\alpha_{B}(V): B \otimes_{F} \mathcal{D}_{B}(V) \rightarrow B \otimes_{\mathbb{Q}_{p}} V .
$$

Fontaine defines $G$-regular $\left(\mathbb{Q}_{p}, G\right)$-rings by certain conditions which insure in particular that $F$ is a field, and that $\alpha_{B}(V)$ is injective for all $V$. Assuming that $B$ is $G$-regular, a representation $V$ is said to be $B$-admissible if $\alpha_{B}(V)$ is an

\footnotetext{
${ }^{13}$ See [Deligne, MR0654325]
} 
isomorphism, or, equivalently, if $\operatorname{dim}_{F} \mathcal{D}_{B}(V)=\operatorname{dim}_{\mathbb{Q}_{p}} V$. When $V$ is $B$-admissible, one says that $V$ and the object $\mathcal{D}_{B}(V)$ of $\mathcal{C}$ are associated (under the correspondence defined by $B$ ).

As a first example, let us consider the Hodge-Tate ring $B_{\mathrm{HT}}=C\left[T, T^{-1}\right]$. On the one hand, it has an obvious graded $K$-algebra structure. On the other hand, it can be endowed with an action of $G$ by setting, for all $g \in G$,

$$
g \cdot\left(\sum_{i \in \mathbb{Z}} \lambda_{i} T^{i}\right)=\sum_{i \in \mathbb{Z}} g\left(\lambda_{i}\right) \chi^{i}(g) T^{i} .
$$

Then $B_{\mathrm{HT}}$ is $\left(\mathbb{Q}_{p}, G\right)$-regular, and a $\mathbb{Q}_{p}$-representation is $B_{\mathrm{HT}^{-}}$ admissible if and only if it is a Hodge-Tate representation as defined in our first section.

The notion of $B$-admissibility can be extended so as to take into account finite extensions of the field $K$ : a $\mathbb{Q}_{p^{-}}$ representation $V$ of $G=\operatorname{Gal}(\bar{K} / K)$ is said to be potentially $B$ admissible if there exists a finite extension $K^{\prime}$ of $K$ in $\bar{K}$ such that $V$ is $B$-admissible as a representation of $\mathrm{Gal}\left(\bar{K} / K^{\prime}\right)$.

\section{Fontaine Rings II: $\mathbf{B}_{\mathrm{dR}}$}

In [7], [8], and [14], Fontaine constructs three new rings which play a central role in his classification of $p$-adic representations: $B_{\mathrm{dR}}, B_{\text {cris }}$ and $B_{\mathrm{st}}$. We now sketch the construction of the biggest one, $B_{\mathrm{dR}}$, which will control the $p$ adic étale cohomology of general algebraic varieties over K.

The first step is to construct a "universal thickening" $A_{\text {inf }}$ of $O_{C}$ [14]. Note that the Frobenius endomorphism $F$ of $O_{C} / p$ is surjective. We denote by $O_{C}^{b}$ the ring defined by

$$
O_{C}^{b}:=\lim _{F} O_{C} / p
$$

If $x=\left(x_{n}\right)_{n \geq 0}$ is an element of $O_{C}^{b}$, and if, for all $n \geq 0$, $\hat{x}_{n} \in O_{C}$ is a lifting of $x_{n}$, then, for all $m \geq 0$, the sequence $\hat{x}_{n+m}^{p^{n}}$ converges towards an element $x^{(m)} \in O_{C}$ when $n \rightarrow \infty$. The elements $x^{(m)}$ do not depend upon the choice of the liftings $\hat{x}_{n}$, and the mapping $\left(x_{n}\right)_{n \geq 0} \mapsto$ $\left(x^{(m)}\right)_{m \geq 0}$ allows one to identify $O_{C}^{b}$ with the set of families $\left(x^{(m)}\right)_{m \geq 0}$ of elements of $O_{C}$ such that $\left(x^{(m+1)}\right)^{p}=x^{(m)}$ for all $m \geq 0$. Using Teichmüller representatives, this provides a $k$-algebra structure on $O_{C}^{b}$.

Fontaine defines a surjective $W$-algebra homomorphism $\theta_{0}: W\left(O_{C}^{b}\right) \rightarrow O_{C}$ by setting, for a Witt vector $\left(x_{n}\right)_{n \geq 0}$ with coordinates in $O_{C}^{b}$,

$$
\theta_{0}\left(\left(x_{n}\right)_{n \geq 0}\right)=\sum_{n \geq 0} p^{n} x_{n}^{(n)} .
$$

He shows that $\theta_{0}$ is an initial object in the category of surjective $W$-algebra homomorphisms $\beta: D \rightarrow O_{C}$, with kernel $I$, such that $D$ and the $D / I^{m+1}$ are $p$-adically separated and complete, and $D \stackrel{\sim}{\rightarrow} \lim _{m} D / I^{m+1}$.
Let $\theta: O_{K} \otimes_{W} W\left(O_{C}^{b}\right) \rightarrow O_{C}$ be the $O_{K}$-linear extension of $\theta_{0}$. The $O_{K}$-algebra $A_{\text {inf }}$ is then defined as the completion of $O_{K} \otimes_{W} W\left(O_{C}^{b}\right)$ for the adic topology defined by the ideal $(p)+\operatorname{Ker}(\theta)$, and one sets $A_{\text {inf, } K}=K \otimes_{O_{K}} A_{\text {inf. }}$.

Keeping the notation $\theta$ for the natural extensions of $\theta$ to $A_{\text {inf }}$ and $A_{\text {inf, }, K}$, one denotes by $J_{K} \subset A_{\text {inf, } K}$ the kernel of $\theta$. It is a maximal principal ideal in $A_{\mathrm{inf}, K}$. One denotes by $B_{\mathrm{dR}}^{+}$the completion of $A_{\mathrm{inf}, K}$ with respect to the $J_{K}$-adic topology. It is a complete discrete valuation ring with residue field $C$. Finally, one defines the ring $B_{\mathrm{dR}}$ as being the fraction field of $B_{\mathrm{dR}}^{+}$.

As all the steps leading to $B_{\mathrm{dR}}$ are compatible with the action of $G$, the algebras $A_{\mathrm{inf}}, A_{\mathrm{inf}, K}, B_{\mathrm{dR}}$ are endowed with an action of $G$. We have $\left(B_{\mathrm{dR}}\right)^{G}=K$, and there is a natural equivariant injection $v: \mathbb{Z}_{p}(1) \hookrightarrow A_{\text {inf }}^{\times}$defined as follows. An element $\varepsilon \in \mathbb{Z}_{p}(1)$ is a sequence $\left(\varepsilon_{n}\right)_{n \in \mathbb{N}}$ such that $\varepsilon_{0}=1$ and $\varepsilon_{n+1}^{p}=\varepsilon_{n}$ for all $n$. It can be viewed as an element of $O_{C}^{b}$ by setting $\varepsilon^{(n)}=\varepsilon_{n}$ for all $n$, and one defines $\nu$ by $\nu(\varepsilon)=1 \otimes[\varepsilon]$, where $[\varepsilon]$ is the Teichmüller representative of $\varepsilon$. Then $\nu(\varepsilon) \in \operatorname{Ker}(\theta)$, and one can define an additive $\operatorname{map} \mathbb{Z}_{p}(1) \rightarrow B_{\mathrm{dR}}^{+}$by

$$
\varepsilon \mapsto \log (\nu(\varepsilon))=\sum_{n \geq 1}(-1)^{n+1}(\nu(\varepsilon)-1)^{n} / n .
$$

Fontaine proved that the image in $B_{\mathrm{dR}}$ of any nonzero element of $\mathbb{Z}_{p}(1)$ is a uniformizing parameter.

The ring $B_{\mathrm{dR}}$ has another fundamental structure: it is endowed with the decreasing filtration defined by its discrete valuation. The Galois action is compatible with this filtration. Thanks to the previous results, one sees that the choice of a nonzero element $t \in \mathbb{Z}_{p}(1)$ provides a $G$ equivariant graded isomorphism

$$
\operatorname{gr}^{\bullet} B_{\mathrm{dR}} \stackrel{\sim}{\rightarrow} \bigoplus_{i \in \mathbb{Z}} C(i)=C\left[t, t^{-1}\right]=B_{\mathrm{HT}},
$$

where $B_{\mathrm{HT}}$ is the previous Hodge-Tate ring.

Following the general pattern, one says that a $\mathbb{Q}_{p^{-}}$ representation $V$ is a de Rham representation if it is $B_{\mathrm{dR}^{-}}$ admissible, i.e., if the canonical map

$$
\alpha_{B_{\mathrm{dR}}}(V): B_{\mathrm{dR}} \otimes_{K}\left(B_{\mathrm{dR}} \otimes_{\mathbb{Q}_{p}} V\right)^{G} \rightarrow B_{\mathrm{dR}} \otimes_{\mathbb{Q}_{p}} V
$$

is an isomorphism. Using the previous results, one gets that de Rham representations are Hodge-Tate.

Remarks. (a) The ring $B_{\mathrm{dR}}$ is functorial with respect to $(K, \bar{K})$. Fontaine shows that if $K^{\prime} \subset \bar{K}$ is a finite extension of $K$, the functoriality map is a filtered isomorphism. As a consequence, $B_{\mathrm{dR}}$ does not depend upon $K$, and has a natural $\bar{K}$-algebra structure.

(b) As $B_{\mathrm{dR}}^{+}$is a complete discrete valuation ring of residue characteristic 0 , it has a (noncanonical) $C$-algebra structure. However, one can show that there does not exist any $G$-equivariant section of the reduction map $B_{\mathrm{dR}}^{+} \rightarrow C$. 
(c) There exist Hodge-Tate representations which are not de Rham.

\section{Fontaine Rings III: $\mathbf{B}_{\text {cris }}$}

The smallest of the three main Fontaine rings, $B_{\text {cris }}$, was introduced at the very beginning of the theory, in order to control the $p$-adic étale cohomology of algebraic varieties with good reduction ${ }^{14}$ over $O_{K}$. There are actually several subrings of $B_{\mathrm{dR}}$ which can be used for that purpose, and the ring $B$ initially used by Fontaine in [7] was later replaced by the ring now known as $B_{\text {cris }}$, better adapted to comparison with crystalline cohomology (see [8], [14]).

A basic tool in crystalline cohomology is the $P D$ envelope of an ideal: given a commutative ring $A$, and an ideal $J \subset A$, there exist a ring homomorphism $\rho: A \rightarrow$ $\mathcal{P}_{A}(J)$ and a $P D$-ideal $\bar{J} \subset \mathcal{P}_{A}(J)$ such that $\rho(J) \subset \bar{J}$, and which are universal for data of this kind (see footnote 9 ).

We return to the setup of the construction of $B_{\mathrm{dR}}$, and we consider again the kernel of the surjective map $\theta_{0}$ : $W\left(O_{C}^{b}\right) \rightarrow O_{C}$. Let $A_{\text {cris }}$ be the $p$-adic completion of the PD-envelope of $\operatorname{Ker}\left(\theta_{0}\right)$. The group $G$ acts by functoriality on $A_{\text {cris }}$. Repeating the construction made for $B_{\mathrm{dR}}$, one gets an equivariant map $\mathbb{Z}_{p}(1) \rightarrow A_{\text {cris }}$. If $t \in A_{\text {cris }}$ is the image of a generator of $\mathbb{Z}_{p}(1)$, one defines $B_{\text {cris }}^{+}=K_{0} \otimes_{W} A_{\text {cris }}$ and $B_{\text {cris }}=B_{\text {cris }}^{+}\left[t^{-1}\right]$. Extending by functoriality the action of $G$ to $B_{\text {cris }}$ one gets $\left(B_{\text {cris }}\right)^{G}=K_{0}$.

From the constructions of $B_{\mathrm{dR}}$ and $B_{\text {cris }}$, one checks that there are natural injective maps

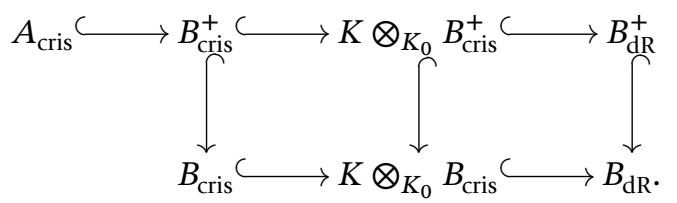

This allows one to endow $K \otimes_{K_{0}} B_{\text {cris }}$ with the filtration induced by the filtration of $B_{\mathrm{dR}}$.

A functoriality argument shows that the Frobenius of $O_{C}^{b}$ defines a Frobenius action $\varphi$ on $A_{\text {cris }} ;$ as $\varphi(t)=p t$, this action extends to $B_{\text {cris }}^{+}$and $B_{\text {cris }}$ and commutes with the $G$ action. Fontaine calls filtered $\varphi$-module the datum of a $K_{0}$ vector space $M_{0}$ endowed with a Frobenius action and a filtration on $K \otimes_{K_{0}} M_{0}$. With these definitions, $B_{\text {cris }}$ has a structure of a filtered $\varphi$-module, compatible with the $G$ action.

A $p$-adic representation $V$ is said to be crystalline if it is $B_{\text {cris }}$-admissible, i.e., if the canonical map

$$
\alpha_{B_{\text {cris }}}(V): B_{\text {cris }} \otimes_{K_{0}}\left(B_{\text {cris }} \otimes_{\mathbb{Q}_{p}} V\right)^{G} \rightarrow B_{\text {cris }} \otimes_{\mathbb{Q}_{p}} V
$$

is an isomorphism. When this is the case, $\alpha_{B_{\text {cris }}}(V)$ is a $G$-equivariant filtered isomorphism, commuting with the Frobenius actions. Moreover, a crystalline representation

\footnotetext{
${ }^{14} i . e .$, which can be extended as a proper and smooth scheme over $O_{K}$.
}

$V$ can be recovered from the associated filtered module $M=\left(B_{\text {cris }} \otimes_{\mathbb{Q}_{p}} V\right)^{G}$ by

$$
V \stackrel{\sim}{\rightarrow} \operatorname{Hom}_{\text {filt. } \varphi \text {-mod. }}\left(K_{0}, B_{\text {cris }} \otimes_{K_{0}} M\right) .
$$

Finally, one sees from the above diagram that crystalline representations are de Rham.

\section{Fontaine Rings IV: $\mathbf{B}_{\text {st }}$}

Using de Jong's theorems [MR1423020], many questions relative to varieties over a discrete valuation field can be reduced to the case of varieties with semistable reduction. It is therefore essential to understand where the cohomology of these varieties stands in Fontaine's classification.

In analogy with the complex and $\ell$-adic cases, Fontaine and Janssen [R1012170] conjectured that the de Rham cohomology of these varieties could be endowed with an additional structure provided by a nilpotent endomorphism, the monodromy operator. This was proved by Hyodo and Kato [MR1293974], and Fontaine introduced a new ring $B_{\mathrm{st}}$ in order to control the cohomology of varieties with semistable reduction.

We will describe $B_{\mathrm{st}}$ as a subring of $B_{\mathrm{dR}}$, which requires us to fix a prime element $\pi \in O_{K}$. Let $s=\left(s_{n}\right)_{n \geq 1}$ be a sequence of elements of $O_{C}$ such that $s_{0}=\pi$ and $s_{n+1}^{p}=s_{n}$ for all $n \geq 0$, and let $\bar{s}_{n} \in O_{C} / p$ be the reduction of $s_{n}$. The sequence $\bar{s}=\left(\bar{s}_{n}\right)_{n \geq 0}$ is an element of $O_{C}^{b}$, and we denote by $\varepsilon(s)=[\bar{s}]$ its Teichmüller representative in $W\left(O_{C}^{b}\right) \subset A_{\text {cris }}$. Then $\varepsilon(s) \pi^{-1} \in 1+$ Fil $^{1} B_{\mathrm{dR}}$, and its logarithm converges in $B_{\mathrm{dR}}^{+}$towards an element $u_{s}$. Fontaine defined $B_{\mathrm{st}}^{+}=B_{\text {cris }}^{+}\left[u_{s}\right], B_{\mathrm{st}}=B_{\text {cris }}\left[u_{s}\right]$. He proved that $u_{s}$ is transcendental over $B_{\text {cris }}^{+}$and that the canonical morphism $K \otimes_{K_{0}} B_{\mathrm{st}} \rightarrow B_{\mathrm{dR}}$ is injective. One endows $K \otimes_{K_{0}} B_{\mathrm{st}}$ with the filtration induced by the filtration of $B_{\mathrm{dR}}$.

These subrings of $B_{\mathrm{dR}}$ are stable under the action of $G$, and we again have $\left(B_{\mathrm{st}}\right)^{G}=K_{0}$. The action of Frobenius on $B_{\text {cris }}$ extends to $B_{\text {st }}$ by setting $\varphi\left(u_{s}\right)=p u_{s}$. In [15], Fontaine defined the monodromy operator $N: B_{\mathrm{st}} \rightarrow B_{\mathrm{st}}$ as being the unique $B_{\text {cris }}$-derivation such that ${ }^{15} N\left(u_{S}\right)=1$. He called filtered $(\varphi, N)$-module the datum of a filtered $\varphi$ module endowed with a $K_{0}$-linear endomorphism $N$ such that $N \varphi=p \varphi N$. In this way, $B_{\mathrm{st}}$ is endowed with a structure of filtered $(\varphi, N)$-module, compatible with the action of $G$. Note that the subrings $B_{\mathrm{st}}^{+}$and $B_{\mathrm{st}}$, as well as $\varphi$ and $N$, depend upon the choice of the prime element $\pi$, but not upon $s$.

As with the previous rings, we will say that a $\mathbb{Q}_{p^{-}}$ representation $V$ is semistable if it is $B_{\mathrm{st}}$-admissible. When $V$ is semistable, the isomorphism $\alpha_{B_{\mathrm{st}}}(V)$ is compatible

${ }^{15}$ To insure a better compatibility with crystalline cohomology, Tsuji uses in MR1705837 the opposite convention $N\left(u_{s}\right)=-1$. 
with all structures, and the representation $V$ can be recovered from the associated filtered module $M$ by

$$
V \stackrel{\sim}{\rightarrow} \operatorname{Hom}_{\text {filt. }(\varphi, N) \text {-mod. }}\left(K_{0}, B_{\text {st }} \otimes_{K_{0}} M\right) .
$$

Semistable representations are de Rham, crystalline representations are semistable, and a semistable representation is crystalline if and only if $N=0$.

\section{Fontaine Conjectures}

As expected, a first, and fundamental, application of Fontaine's theory is a classification of $p$-adic representations. In contrast with $\ell$-adic representations of $G_{K}(\ell \neq p)$, whose structure is rather simple, ${ }^{16} p$-adic representations of $G_{K}$ have a complicated structure: for example, even when a variety $X$ over $K$ has good reduction, the inertia group may act wildly on $H_{\text {ét }}\left(X_{\bar{K}}, \mathbb{Q}_{p}\right)$, while it acts trivially in the $\ell$-adic case, $\ell \neq p$. Until Fontaine set up his formalism of $B$ rings, the understanding of this wildness and a classification of $p$-adic representations of $G_{K}$ seemed hopeless. Using Fontaine's rings $B_{\mathrm{cris}}, B_{\mathrm{st}}, B_{\mathrm{dR}}, B_{\mathrm{HT}}$, and the corresponding $B$-admissibility and potential $B$-admissibility conditions, we now have a hierarchy (with a "?" discussed as Conjecture/Theorem 6):

$$
\begin{array}{ccccc}
\text { crystalline } & \Rightarrow & \text { semistable } & \Rightarrow & \text { de Rham } \\
\Downarrow & & \Downarrow & ? & \| \\
\text { pot. cryst. } & \Rightarrow & \text { pot. semist. } & \stackrel{?}{\Leftrightarrow} & \text { de Rham } \\
& & & \begin{array}{c}
\Downarrow \\
\end{array} & \\
& & & \text { Hodge-Tate }
\end{array}
$$

Hints for this classification came of course from geometry. Fontaine expected that the geometric properties of an algebraic variety $X$ over $K$ would be reflected in the position of its $p$-adic étale cohomology spaces in this hierarchy, and he made a series of conjectures to that effect.

Assume only that $X$ is proper and smooth over $K$. Fontaine made the following conjecture [7]:

Conjecture/Theorem $2\left(\mathbf{C}_{\mathbf{d R}}\right)$. Under the previous assumptions, $H_{\mathrm{et}}^{m}\left(X_{\bar{K}}, \mathbb{Q}_{p}\right)$ is de Rham, and associated with $H_{\mathrm{dR}}^{m}(X / K)$ endowed with its Hodge filtration; i.e., there exists a canonical isomorphism

$$
B_{\mathrm{dR}} \otimes_{K} H_{\mathrm{dR}}^{m}(X / K) \stackrel{\sim}{\rightarrow} B_{\mathrm{dR}} \otimes_{\mathbb{Q}_{p}} H_{\mathrm{et}}^{m}\left(X_{\bar{K}}, \mathbb{Q}_{p}\right),
$$

compatible with filtrations and Galois actions on both sides.

Using the results of section 5 , one sees that $\mathbf{C}_{\mathbf{d R}}$ implies $\mathbf{C}_{\mathrm{HT}}$.

Assume now that $X / K$ has good reduction, and let $X$ be a proper and smooth $O_{K}$-scheme such that $X=x_{K}$. By a

\footnotetext{
${ }^{16}$ By Grothendieck's local monodromy theorem, when no finite extension of $k$ contains all the roots of unity of order a power of $\ell$, the action of the inertia group is quasi-unipotent.
}

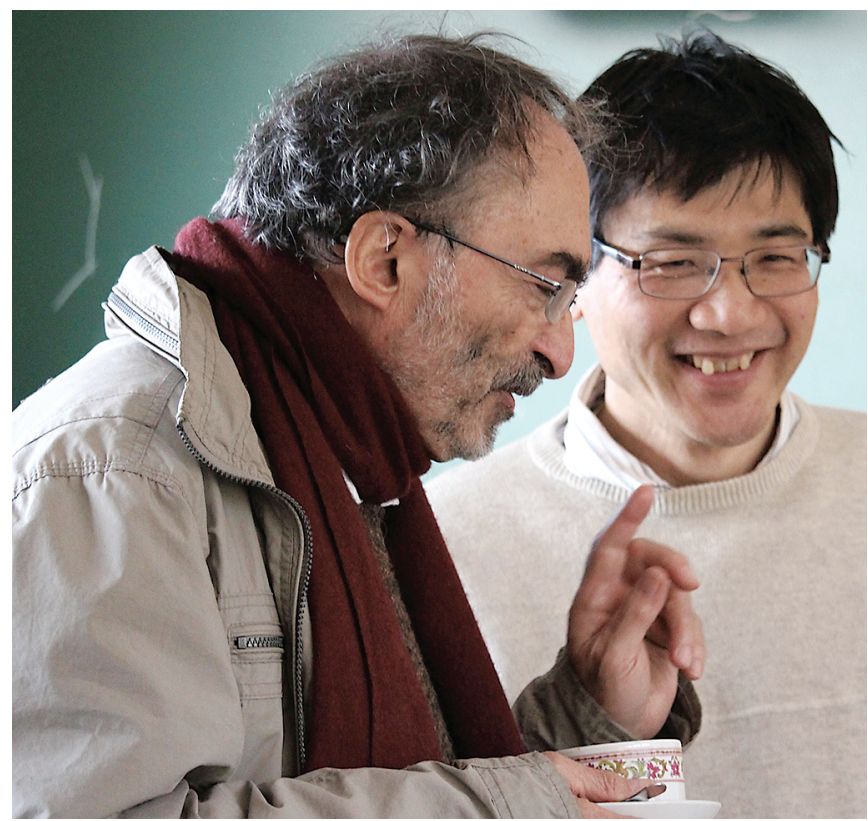

Figure 5. Jean-Marc Fontaine making his point with Takeshi Saito.

theorem of Berthelot-Ogus [MR0700767], there is a canonical isomorphism

$$
K \otimes_{W} H_{\text {cris }}^{m}\left(x_{k} / W\right) \stackrel{\sim}{\rightarrow} H_{\mathrm{dR}}^{m}(X / K)
$$

where $H_{\mathrm{cris}}^{m}\left(X_{k} / W\right)$ is the crystalline cohomology of the special fiber $\mathcal{X}_{k}$. This endows the $K_{0}$-vector space $K_{0} \otimes$ $H_{\text {cris }}^{m}\left(X_{k} / W\right)$ with a structure of filtered $\varphi$-module. Combining this structure with the analogous one on $B_{\text {cris }}$ Fontaine made the following conjecture [7]:

Conjecture/Theorem $3\left(\mathbf{C}_{\text {cris }}\right)$. With the above notation, $H_{\mathrm{et}}^{m}\left(X_{\bar{K}}, \mathbb{Q}_{p}\right)$ is crystalline, and associated with $K_{0} \otimes_{W}$ $H_{\mathrm{cris}}^{m}\left(\mathcal{X}_{k} / W\right)$; i.e., there exists a canonical isomorphism

$$
B_{\text {cris }} \otimes_{W} H_{\text {cris }}^{m}\left(x_{k} / W\right) \stackrel{\sim}{\rightarrow} B_{\text {cris }} \otimes_{\mathbb{Q}_{p}} H_{\text {et }}^{m}\left(X_{\bar{K}}, \mathbb{Q}_{p}\right)
$$

compatible with Frobenius and Galois actions on both sides, and with the filtrations after extension to $B_{\mathrm{dR}}$.

In the semistable reduction case, where $X=x_{K}$ for a proper semistable $O_{K}$-scheme $\mathcal{X}$, Hyodo and Kato used the theory of logarithmic structures initiated by Fontaine and Illusie, and developed by Kato and many others, to replace the crystalline cohomology of $X_{k}$ by its $\log$ crystalline cohomology. Generalizing the aforementioned theorem of Berthelot-Ogus, they defined a canonical isomorphism $K \otimes_{W} H_{\text {logcris }}^{m}\left(x_{k} / W\right) \stackrel{\sim}{\rightarrow} H_{\mathrm{dR}}^{m}(X / K)$ (see [MR1293974]) which endows $K_{0} \otimes H_{\text {logcris }}^{m}\left(X_{k} / W\right)$ with a structure of filtered $(\varphi, N)$-module. Combining this structure with the analogous one on $B_{\text {st }}$, Fontaine and Jannsen made the following conjecture ([11], [MR1293975]): 
Conjecture/Theorem $4\left(\mathbf{C}_{\text {st }}\right)$. With the above notation, $H_{\mathrm{e} t}^{m}\left(X_{\bar{K}}, \mathbb{Q}_{p}\right)$ is semistable, and associated with $K_{0} \otimes_{W}$ $H_{\log c r i s}^{m}\left(X_{k} / W\right)$; i.e., there exists a canonical isomorphism

$$
B_{\mathrm{st}} \otimes_{W} H_{\log \text { cris }}^{m}\left(X_{k} / W\right) \stackrel{\sim}{\rightarrow} B_{\mathrm{st}} \otimes_{\mathbb{Q}_{p}} H_{\mathrm{et}}^{m}\left(X_{\bar{K}}, \mathbb{Q}_{p}\right)
$$

compatible with $\varphi, N$, and Galois actions on both sides, and with the filtrations after extension to $B_{\mathrm{dR}}$.

By now conjectures $\mathbf{C}_{\mathbf{d R}}, \mathbf{C}_{\text {cris }}$, and $\mathbf{C}_{\mathbf{s t}}$ (and in particular $\mathbf{C}_{\mathbf{H T}}$ ) have been fully proved. Actually, $\mathbf{C}_{\text {cris }}$ turns out to be a particular case of $\mathbf{C}_{\text {st }}$ (with $N=0$ ), and it was shown, using de Jong's alterations, that $\mathbf{C}_{\mathrm{st}}$ implies $\mathbf{C}_{\mathbf{d R}}$. Conjecture $\mathbf{C}_{\text {st }}$ was first proved by Tsuji [MR1705837].

The history of the proofs of these conjectures is long and involves many mathematicians. Bloch and Kato had proved $\mathbf{C}_{\mathbf{H T}}$ in the case of good ordinary reduction MR0849653. However their method did not extend to the general case, and gave no idea on how to attack $\mathbf{C}_{\text {cris }}$. The first breakthrough was made by Fontaine and Messing [10], who proved $\mathbf{C}_{\text {cris }}$ for $K=K_{0}$ and $\operatorname{dim} X<p$. For this they introduced a new and fruitful technique, that of syntomic sheaves and complexes. Kato's proof of $\mathbf{C}_{\mathbf{s t}}$ (under certain restrictions of dimension) and Tsuji's proof (in the general case) basically follow Fontaine-Messing's method. At about the same time as Fontaine-Messing's work, Faltings developed quite a different approach, based on the theory of almost étale extensions, a far-reaching generalization of some fundamental results of Tate in [MR0231827]. A flaw was found in his first proofs of $\mathbf{C}_{\mathbf{d R}}$ and $\mathbf{C}_{\text {cris }}$, which he corrected in [MR1922831], giving a new proof of $\mathbf{C}_{\mathbf{s t}}$. Finally, Niziol [MR2372150] proved $\mathbf{C}_{\text {st }}$ by still another method, based on higher $K$-theory, and showed that the comparison isomorphisms established by all these different methods were actually the same. More recently, Beilinson gave simple proofs of these isomorphisms, by a totally different approach, using techniques of derived algebraic geometry (see [MR2904571], MR3272051]).

\section{More Conjectures}

In addition to these conjectures, the development of Fontaine's theory has raised some new questions involving the structures used in his classification of $\mathbb{Q}_{p}$-representions. The solutions of the next two conjectures have been very important steps in $p$-adic Hodge theory.

9.1. Weak admissibility. From the very beginning, Fontaine wanted to give an intrinsic characterization of the filtered $(\varphi, N)$-modules that could be associated with a crystalline (and, later on, semistable) representation, calling them in short "admissible modules." He investigated the Newton and Hodge polygons defined by the Frobenius action and the filtration of such a module, and he discovered a remarkable property, reminiscent of the semistability condition for vector bundles on curves: these polygons have the same terminal points, and, for any submodule, Newton is above Hodge. He called the filtered $(\varphi, N)$-modules satisfying this property weakly admissible, and he made the following conjecture ([5], [15]):

Conjecture/Theorem 5. Weakly admissible $(\varphi, N)$-modules are admissible.

This conjecture resisted all attempts for over twenty years. Exploiting the analogy between weak admissibility and semistability, Faltings proved a partial result in this direction, namely, the stability (in the crystalline case) of weak admissibility under tensor product [MR1403965. The semistable case was then treated by Totaro [MR1388844]. Conjecture 5 was eventually proved in 2000 by Colmez and Fontaine [MR1779803]. More recently, Fargues and Fontaine gave a beautiful new proof, making the analogy become a true correspondence (see section 12).

9.2. Potentially semistable representations. As semistable representations are de Rham, and $B_{\mathrm{dR}}$ does not change if $K$ is replaced by a finite extension contained in $C$, potentially semistable representations are also de Rham. However, no example was known of a de Rham representation that would not be potentially semistable, which is symbolized by the "?" in the hierarchy displayed in section 8. This led Fontaine to the conjecture [15]:

Conjecture/Theorem $6\left(\mathbf{C}_{\mathrm{pst}}\right)$. De Rham representations are potentially semistable.

This conjecture was hard, too. Several proofs were (independently) given around 2002, by André [MR1906151], Kedlaya |MR2119719|, and Mebkhout [MR1906152|, using new ingredients: the theory of $(\varphi, \Gamma)$-modules (another fundamental contribution of Fontaine [12]; see the end of section 10), and that of $p$-adic differential equations over the Robba ring (see [Berger, MR1906150|). A simplified proof was later given by Colmez [MR2493217].

\section{Impact on Arithmetic Geometry}

Fontaine's work has rapidly found deep applications to algebraic geometry. A beautiful example was given by his proof of a conjecture of Shafarevich: a good control of the ramification of finite commutative group schemes over $\mathbb{Z}$ allowed him to show that there is no nonzero abelian scheme over Spec $\mathbb{Z}$ [9] (the conjecture was also proved, independently, by Abrashkin). In the same vein, the full force of Fontaine's theory served as a crucial ingredient in many global results and conjectures over number fields. Let us present here very briefly a few examples.

10.1. The Bloch-Kato conjecture on special values of $L$-functions. Let $k$ be a number field, and let $V$ be the 
$p$-adic realization of a Chow motive $M$ over $k$, e.g., $V=$ $H^{m}\left(X_{\bar{k}}, \mathbb{Q}_{p}\right)$ for $X$ projective and smooth over $k$. Using the rings $B_{\text {cris }}$ associated with the places of $k$ over $p$, Bloch and Kato define in [MR1086888] a subspace

$$
H_{f}^{1}(k, V) \subset H^{1}(k, V),
$$

from which they in turn define a subgroup

$$
H_{f}^{1}(k, M) \subset H^{1}(k, M)
$$

of a motivic cohomology group, closely related to a TateShafarevich group when $M$ is the motive of an abelian variety over $k$. The Bloch-Kato conjecture predicts the order of vanishing of $L(M, s)$ at $s=0$ as $\operatorname{dim} H_{f}^{1}\left(k, M^{\vee}(1)\right)-$ $\operatorname{dim} H^{0}\left(k, M^{\vee}(1)\right)$ and determines the leading coefficient of the Taylor series expansion of $L(M, s)$ at zero, by a formula generalizing the Birch and Swinnerton-Dyer conjecture. An equivariant version, due to Kato, plays an important role in Iwasawa theory. See [MR1086888], [13].

10.2. Serre's modularity conjecture. The following conjecture is due to Serre [MR0885783]:

Conjecture/Theorem 7. Let $r: G_{\mathbb{Q}}=\mathrm{Gal}(\overline{\mathbb{Q}} / \mathbb{Q}) \rightarrow \mathrm{GL}_{2}(F)$ be a continuous, irreducible, and odd representation, where $F$ is a finite field of characteristic $p$. Then $r$ is modular, i.e., is the reduction modulo the maximal ideal of the ring of integers $\mathcal{O}$ of a finite extension of $\mathbb{Q}_{p}$ of the representation $\rho_{f}: G_{\mathbb{Q}} \rightarrow \mathrm{GL}_{2}(\mathcal{O})$ associated with a newform $f \in S_{k}\left(\Gamma_{1}(N)\right)$, for a suitable weight $k$ and level $N$ coprime to $p$.

The precise conjecture prescribes $k$ and $N$ in terms of $r$. It is known that this conjecture implies the ShimuraTaniyama-Weil modularity conjecture for elliptic curves over $\mathbb{Q}$, proved by Wiles, Taylor-Wiles et al. Conjecture 7 has been proved by Khare and Wintenberger [MR2551764, modulo some hypothesis, later proved by Kisin [MR2551765.

The proof heavily relies on modularity lifting theorems due to Kisin [MR2600871] et al. for $p$-adic representations of $G_{\mathbb{Q}}$ with prescribed ramification at $p$, expressed in terms of Fontaine's classification (crystalline, semistable, potentially semistable).

10.3. The Fontaine-Mazur conjecture. Let $k$ be a number field, and let $p$ be a prime number. Let $G_{k}=\operatorname{Gal}(\bar{k} / k)$ be the absolute Galois group of $k$. Let $X / k$ be proper and smooth and let $m \in \mathbb{Z}$. The $p$-adic representation $H_{\mathrm{et}}^{m}\left(X_{\bar{k}}, \mathbb{Q}_{p}\right)$ of $G_{k}$ is unramified oustside a finite set of places of $k$, and, by the $p$-adic comparison Theorem 3 (together with 6), is potentially semistable at any prime above $p$. The same is true of any subquotient. Fontaine and Mazur conjectured the following converse [16]:

Conjecture/Theorem 8. Let $\rho: G_{k} \rightarrow \mathrm{GL}_{n}\left(\mathbb{Q}_{p}\right)$ be a continuous, irreducible representation which is unramified outside a finite number of places, and potentially semistable at every place above $p$. Then $\rho$ is a subquotient of a representation of the form $H^{m}\left(X_{\bar{k}}, \mathbb{Q}_{p}\right)$ for some proper and smooth $X / k$ and $m \in \mathbb{Z}$.

The case $n=1$ had been known for a long time. For $n=2$ and $k=\mathbb{Q}$, Conjecture 8 takes the more precise form:

Conjecture/Theorem 9. Let $\rho: G_{\mathbb{Q}} \rightarrow \mathrm{GL}_{2}\left(\mathbb{Q}_{p}\right)$ be a continuous representation. Assume that $\rho$ is irreducible, odd, unramified outside a finite number of primes, and potentially semistable at $p$. Then there exists $i \in \mathbb{Z}$ and a newform $f$ as above such that $\rho$ is isomorphic to $\rho_{f} \otimes \chi_{p}^{i}$, where $\chi_{p}: G_{\mathbb{Q}} \rightarrow \mathbb{Z}_{p}^{*}$ is the cyclotomic character.

Conjecture 9 has been proved independently by Emerton [MR2251474] and by Kisin (with some mild technical restrictions [MR2505297]). In addition to Kisin's theory of potentially semistable deformation rings (|MR2373358, [MR2827797]), the proofs use a deep, new ingredient, a p-adic local Langlands correspondence between absolutely irreducible 2-dimensional $p$-adic representations of $G_{\mathbb{Q}_{p}}=$ $\operatorname{Gal}\left(\overline{\mathbb{Q}_{p}} / \mathbb{Q}_{p}\right)$ and certain absolutely irreducible unitary Banach representations of $\mathrm{GL}_{2}\left(\mathbb{Q}_{p}\right)$, constructed by Breuil in some cases, and by Breuil and Colmez in general (see [MR2906353] and Astérisque 319, 330, 331). Their construction heavily relies on Fontaine's theory of $(\varphi, \Gamma)$-modules. This theory, introduced by Fontaine [12], and developed by Berger, Cherbonnier, Colmez, and Kedlaya, describes $p$-adic representations of $G_{\mathbb{Q}_{p}}$ in terms of modules over power series rings in one variable over $\mathbb{Q}_{p}$ (or a finite extension) equipped with certain operators (a "Frobenius" $\varphi$ and an action of a group $\Gamma$ isomorphic to $\mathbb{Z}_{p}^{*}$ ).

\section{Norm Fields and Tilts}

Spectacular generalizations and applications of Fontaine's theory have been developed by Peter Scholze. His theory of perfectoid spaces has its roots in:

(a) Fontaine's construction of the $B$ rings, as seen above;

(b) Fontaine-Wintenberger's theory of norm fields.

These constructions make sense for complete, valuative extensions $L$ of $K$ of height one such that the Frobenius endomorphism of $O_{L} / p$ is surjective. In ([4], [MR0719763]), Fontaine and Wintenberger constructed, for a large class of infinite extensions $E$ of $K$ (called arithmetically profinite), a field $\widetilde{E}$ of characteristic $p$, called the norm field of $E$, with the remarkable property that the absolute Galois groups of $E$ and $\widetilde{E}$ are canonically isomorphic. Moreover, if $L$ is the $p$-adic completion of $E$, the fraction field $L^{b}$ of $O_{L}^{b}$ is the completion of the radicial closure of $\widetilde{E}$ (hence the absolute Galois groups of $L$ and $L^{b}$ are also canonically isomorphic). As an example, for $K=\mathbb{Q}_{p}, E=\mathbb{Q}_{p}\left(p^{1 / p^{\infty}}\right)$, we have 
$\widetilde{E}=\mathbb{F}_{p}((t))$, and the absolute Galois groups of $\mathbb{Q}_{p}\left(p^{1 / p^{\infty}}\right)$ and $\mathbb{F}_{p}((t))$ are canonically isomorphic.

Scholze called fields such as $L$ perfectoid, and $L^{b}$ the tilt of $L$. It was the starting point of his theory of perfectoid spaces, which has had several striking applications outside of the $p$-adic world, e.g., the proof of the weight monodromy conjecture in $\ell$-adic cohomology in mixed characteristic for complete intersections in the projective space |MR3090258.

\section{The Fargues-Fontaine Curve}

In the first decade of this century, most of the conjectures that fueled the development of $p$-adic Hodge theory had been proved, and the landscape of Fontaine's rings was looking almost familiar. One could then have thought that the theory had essentially reached its final shape. But that would have been ignoring how much Fontaine liked to change perspective and develop new points of view on everything, including his own work.

So, in a joint work with Laurent Fargues, he surprised everyone again. In [18], [19] (considerably amplified in [20]), they constructed and studied a Noetherian scheme $X$ over $\mathbb{Q}_{p}$, regular of dimension 1 , now called the FarguesFontaine curve. This geometric object, obtained by gluing pieces of Fontaine's ring $B_{\text {cris }}$, sheds a totally new light on $p$-adic Hodge theory.

Though not being of finite type over $\mathbb{Q}_{p}$, it resembles the projective line over $\mathbb{Q}_{p}: H^{0}(X, \mathcal{O})=\mathbb{Q}_{p}$, and every vector bundle over $X$ is a sum of line bundles. If $K$ is a finite extension of $\mathbb{Q}_{p}$, the absolute Galois group $G_{K}=$ $\operatorname{Gal}(\bar{K} / K)$ acts on $X$, and Fargues and Fontaine establish an equivalence of categories between $\mathbb{Q}_{p}$-representations of $G_{K}$ and $G_{K}$-equivariant vector bundles on $X$ which are semistable of slope zero. This leads to simpler, more conceptual proofs of Conjectures 5 and 6 . But there is a much deeper potential application, namely, the Fargues geometrization conjecture for the local Langlands correspondence [arXiv:1602.00999], intensely studied today with Scholze's theory of diamonds [arXiv:1709.07343].

\section{To Conclude...}

Solving the problem of the "mysterious functor" was one of the biggest challenges left by Grothendieck in the early seventies. To that effect, Jean-Marc Fontaine patiently and systematically developed a program which unlocked a whole new area of number theory and algebraic geometry, apparently out of reach before his work. He has been a leading force in the subject during his whole life, constantly enriching it with new methods, results, and conjectures. He was always ready to share his ideas, and several of his former students have been themselves major contributors to the development of $p$-adic Hodge theory. Jean-Marc Fontaine has left us now, but he will still be present among us for a very long time, thanks to the richness of his mathematical heritage.

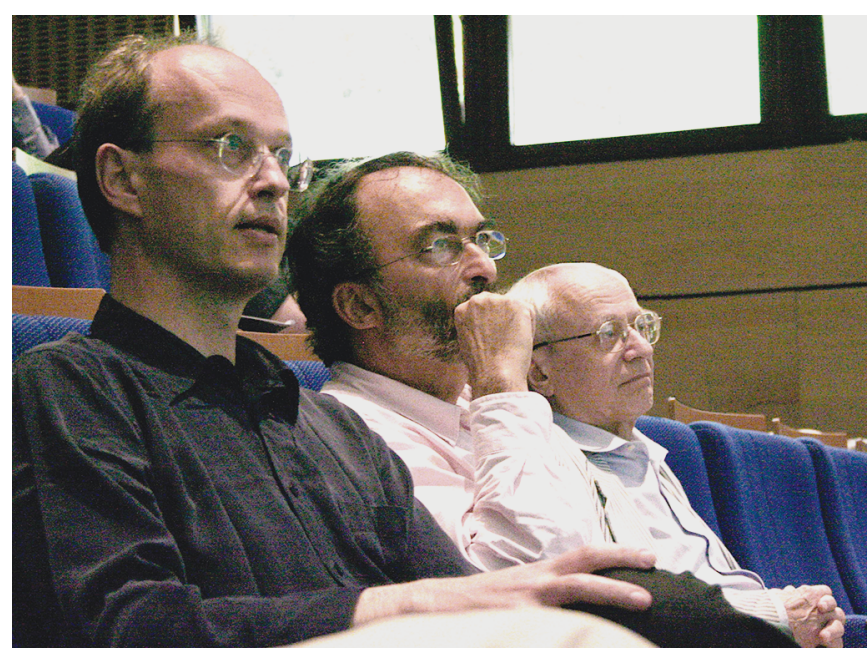

Figure 6. Jean-Marc Fontaine attending a lecture with Pierre Colmez and Jean-Pierre Serre.

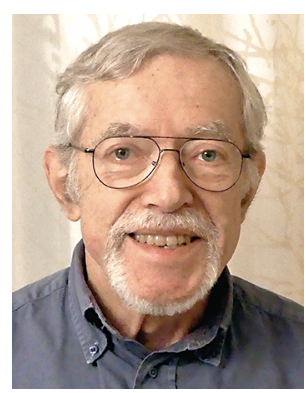

Pierre Berthelot

\section{Fontaine's Rings and Geometry}

\section{Peter Scholze}

One of the most striking results of Fontaine is his theory of the field of norms, developed jointly with his student Jean-Pierre Wintenberger. While the field

$$
\mathbb{Q}_{p}=\left\{\sum_{n \gg-\infty} a_{n} p^{n} \mid a_{n} \in\{0,1, \ldots, p-1\}\right\}
$$

of $p$-adic numbers and the field

$$
\mathbb{F}_{p}((t))=\left\{\sum_{n \gg-\infty} a_{n} t^{n} \mid a_{n} \in\{0,1, \ldots, p-1\}=\mathbb{F}_{p}\right\}
$$

Peter Scholze is a professor of mathematics at the Mathematisches Institut, Universität Bonn, and Max-Planck-Institut für Mathematik, Bonn, Germany. His email address is scholze@math. uni-bonn. de. 
share many formal similarities, there is no direct algebraic relationship. For example the absolute Galois group of $\mathbb{Q}_{p}$ is topologically finitely generated, while that of $\mathbb{F}_{p}((t))$ is not. However:

Theorem (Fontaine-Wintenberger, [4]). Consider the fields $K_{n}=\mathbb{Q}_{p}\left(\zeta_{p^{n}}\right)$ obtained from $\mathbb{Q}_{p}$ by adjoining a primitive $p^{n}$ th root of unity $\zeta_{p^{n}}$, and let $K_{\infty}=\bigcup K_{n}$ be their compositum. The absolute Galois groups of $K_{\infty}$ and $\mathbb{F}_{p}((t))$ are canonically isomorphic.

The result holds for many other infinite extensions of $\mathbb{Q}_{p}$, e.g., the one obtained by adjoining all $p$-power roots of $p$. Contemplating this result was a main inspiration for the theory of perfectoid spaces. While I was working on these results, still a PhD student, I was given the opportunity to present this work at a course at the IHES in the fall of 2011. It was an enormous honor that Fontaine attended these lectures, and the support he expressed was most significant to me.

In this note, I want to focus however on another object introduced by Fontaine: his ring of $p$-adic periods $B_{\mathrm{dR}}^{+}$. Let me give a brief summary of its character.

The ring $B_{\mathrm{dR}}^{+}$is a complete discrete valuation ring whose residue field is the field $\mathbb{C}_{p}$, the completion of $\overline{\mathbb{Q}}_{p}$. Moreover, it has a natural topology, inducing the usual $p$-adic topology on $\mathbb{C}_{p}$, and a natural action of the absolute Galois group $G_{\mathbb{Q}_{p}}$.

Note that by Cohen's structure theorem, there is an isomorphism of $B_{\mathrm{dR}}^{+}$with a power series ring over $\mathbb{C}_{p}$, as complete discrete valuation rings whose residue field $\mathbb{C}_{p}$ is of characteristic $0:{ }^{17}$

$$
B_{\mathrm{dR}}^{+} \cong \mathbb{C}_{p}[[t]] .
$$

However, there is no such isomorphism that is continuous, and no such isomorphism that is Galois-equivariant.

The original motivation for introducing the ring $B_{\mathrm{dR}}^{+}$ (and for its name, the "ring of $p$-adic (de Rham) periods") is the role that it plays in $p$-adic Hodge theory. Recall that if $X$ is a smooth complex variety, then there is a natural isomorphism

$$
H^{i}(X, \mathbb{Z}) \otimes_{\mathbb{Z}} \mathbb{C} \cong H_{\mathrm{dR}}^{i}(X)
$$

between singular and de Rham cohomology. On a concrete level, this means that one has a pairing between differential forms and cycles; e.g., if $X=\mathbb{C}^{\times}$, one can take $\omega=\frac{d z}{z}$ and the circle $\gamma=S^{1} \subset \mathbb{C}^{\times}$to get the period

$$
\int_{S^{1}} \frac{d z}{z}=\int_{0}^{1} \frac{d\left(e^{2 \pi i t}\right)}{e^{2 \pi i t}}=\int_{0}^{1} 2 \pi i d t=2 \pi i \in \mathbb{C} .
$$

\footnotetext{
${ }^{17}$ From what I hear, it took several decades for the mathematical community to understand the significance of $B_{\mathrm{dR}^{\prime}}^{+}$not least because it's just $\mathbb{C}_{p}[[t]]$ after all!
}

Similarly, a conjecture of Fontaine, known as $\left(C_{\mathrm{dR}}\right),{ }^{18}$ asserts that for a smooth variety $X$ over $\mathbb{Q}_{p}$, there is a natural isomorphism

$$
H^{i}\left(X_{\mathbb{C}_{p}}, \mathbb{Z}_{p}\right) \otimes_{\mathbb{Z}_{p}} B_{\mathrm{dR}} \cong H_{\mathrm{dR}}^{i}(X) \bigotimes_{\mathbb{Q}_{p}} B_{\mathrm{dR}}
$$

between étale and de Rham cohomology. Here $B_{\mathrm{dR}}$ is the fraction field of $B_{\mathrm{dR}}^{+}$. At a concrete level, this means that given a differential form and a cycle, one can get periods in $B_{\mathrm{dR}}$. This can be applied to analogues of $\omega=\frac{d z}{z}$ and $\gamma$ as above, leading in that case to an element $t \in B_{\mathrm{dR}}^{+}$that is in fact a uniformizer of $B_{\mathrm{dR}}^{+}$. This is the so-called " $p$-adic analogue of $2 \pi i$." In particular, the periods thus obtained do not lie in $\mathbb{C}_{p}$.

Now I claim that by defining $B_{\mathrm{dR}^{\prime}}^{+}$, Fontaine was the first person to get a glimpse of a part of the elusive "arithmetic surface"

\section{$\operatorname{Spec} \mathbb{Z} \times \operatorname{Spec} \mathbb{Z}$.}

Recall that in algebraic geometry, there is a space $\operatorname{Spec} A$ associated with any commutative ring $A$. For the simplest (namely, initial) ring $A=\mathbb{Z}$ of the integers, the points of Spec $\mathbb{Z}$ are exactly the prime numbers $2,3,5,7,11, \ldots$, plus a generic point $\eta$. Any other $\operatorname{ring} A$ admits a unique map $\mathbb{Z} \rightarrow A$, which induces a map $\operatorname{Spec} A \rightarrow \operatorname{Spec} \mathbb{Z}$. In other words, all of algebraic geometry lives over the base space Spec $\mathbb{Z}$.

However, as emphasized for example by Weil, in many respects $S p e c \mathbb{Z}$ behaves like a curve, in fact very much like the affine line Spec $\mathbb{F}_{p}[T]$ over a finite field $\mathbb{F}_{p}$. The theorem of Fontaine-Wintenberger is one of the most profound manifestations of this analogy. In the arithmetic of Spec $\mathbb{F}_{p}[T]$, a critical role is played by the arithmetic surface

$$
\operatorname{Spec} \mathbb{F}_{p}[T] \times_{\mathbb{F}_{p}} \operatorname{Spec} \mathbb{F}_{p}[T]=\operatorname{Spec} \mathbb{F}_{p}\left[T_{1}, T_{2}\right] \text {. }
$$

In particular, Weil's proof of his conjectures for curves is based on intersecting curves on this surface.

Unfortunately, no such space is available for $\operatorname{Spec} \mathbb{Z}$, precisely because all objects in algebraic geometry come with a unique map to $\operatorname{Spec} \mathbb{Z}$. It has long been dreamt that there is still some way to make sense of

\section{$\operatorname{Spec} \mathbb{Z} \times \operatorname{Spec} \mathbb{Z}$,}

but it is hard to fill this dream with interesting mathematics-taken inside schemes, the best one can do is $\operatorname{Spec} \mathbb{Z} \times_{\text {Spec } \mathbb{Z}} \operatorname{Spec} \mathbb{Z}$, which is just Spec $\mathbb{Z}$ itself. Dreaming on for a moment, there should be a diagonal embedding

$$
\Delta: \operatorname{Spec} \mathbb{Z} \hookrightarrow \operatorname{Spec} \mathbb{Z} \times \operatorname{Spec} \mathbb{Z},
$$

and the completion of Spec $\mathbb{Z} \times \operatorname{Spec} \mathbb{Z}$ along $\Delta$ should be some 1-parameter deformation of Spec $\mathbb{Z}$. More generally, for any ring $A$, there should be an object $\operatorname{Spec} A \times \operatorname{Spec} \mathbb{Z}$,

\footnotetext{
${ }^{18}$ First proved by Tsuji, with now many different proofs known.
} 
and the natural map $\operatorname{Spec} A \rightarrow \operatorname{Spec} \mathbb{Z}$ should induce a graph

$$
\Gamma_{A}: \operatorname{Spec} A \hookrightarrow \operatorname{Spec} A \times \operatorname{Spec} \mathbb{Z} .
$$

The completion of $\operatorname{Spec} A \times \operatorname{Spec} \mathbb{Z}$ should be a natural 1parameter deformation of $A$.

This is precisely what Fontaine has defined in the case of $A=\mathbb{C}_{p}$ ! Indeed $\operatorname{Spec} B_{\mathrm{dR}}^{+} \simeq \operatorname{Spec} \mathbb{C}_{p}[[t]]$ is a certain canonical 1-parameter deformation of $\operatorname{Spec} \mathbb{C}_{p}$.

In joint work with Fargues on the Fargues-Fontaine curve, [20], Fontaine has actually gone further and has arguably described all of Spec $\mathbb{C}_{p} \times \operatorname{Spec} \mathbb{Z}_{p}$, making it into an actual mathematical object. By leaving the diagonal $\Delta(\operatorname{Spec} \mathbb{Z}) \subset \operatorname{Spec} \mathbb{Z} \times$ Spec $\mathbb{Z}$ near a prime $p$, Fontaine has led us into a whole new and mysterious world.

This was actually the motivation for my work on "diamonds," about which I have also given a course at the IHES in March/April 2017. At the time, Fontaine had already been on cancer treatment. It was a very pleasant surprise, and again a deep honor, that Fontaine was able to attend the talks. In fact, I was very happy to see him in good spirits, and as energetic as ever in his mathematics. His passion for mathematics was truly inspiring.

Where will his mathematics lead us? In joint work with Clausen, on January 24, 2019, we found an analogue of the ring $B_{\mathrm{dR}}^{+}$for the real numbers $\mathbb{R}$. Again, it appears to be just $\mathbb{R}[[t]]$ at first sight. However, we are working with the notion of what we termed an analytic ring, which is roughly a topological ring $R$ equipped with a notion of $R$ valued measures on any profinite set $S$. It turns out that for $0<p<1$, the space of " $p$-measures" $\mathcal{M}_{p}(S, \mathbb{R})$ with real coefficients has a canonical nonsplit self-extension, which may be called $\mathcal{M}_{p}\left(S, B_{\mathrm{dR}}^{+}(\mathbb{R}) / t^{2}\right)$. It comes from the entropy function and some avatars of it had been known in the functional analysis literature. We realized that this nonsplit self-extension could even be put into an infinite tower, leading to spaces of measures $\mathcal{M}_{p}\left(S, B_{\mathrm{dR}}^{+}(\mathbb{R})\right)$, which have a ring structure in the suitable sense. Moreover, the definition of $B_{\mathrm{dR}}^{+}(\mathbb{R})$ is similar to Fontaine's definition, as a certain completion of a ring generated by "Teichmüller representatives" of the reals.

I immediately wondered whether I should inform Fontaine that his ideas had now truly become real; however, I first wanted to be sure that these ideas were correct. Fontaine died five days later, on January 29, 2019.

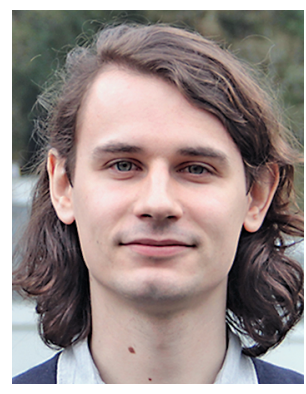

Peter Scholze

\section{References}

[1] Jean-Marc Fontaine, Extensions finies galoisiennes des corps valués complets à valuation discrète (French), Séminaire Delange-Pisot-Poitou: 1967/68, Théorie des Nombres, Secrétariat mathématique, Paris, 1969, Fasc. 1, Exposé 6, 21 pp. MR0246857

[2] Jean-Marc Fontaine, Groupes de ramification et représentations d'Artin (French), Ann. Sci. École Norm. Sup. (4) 4 (1971), 337-392. MR289458

[3] Jean-Marc Fontaine, Groupes p-divisibles sur les corps locaux (French), Astérisque, No. 47-48, Société Mathématique de France, Paris, 1977. MR0498610

[4] Jean-Marc Fontaine and Jean-Pierre Wintenberger, Le "corps des normes" de certaines extensions algébriques de corps locaux (French, with English summary), C. R. Acad. Sci. Paris Sér. A-B 288 (1979), no. 6, A367-A370. MR526137

[5] Jean-Marc Fontaine, Modules galoisiens, modules filtrés et anneaux de Barsotti-Tate (French), Journées de Géométrie Algébrique de Rennes (Rennes, 1978), Astérisque, vol. 65, Soc. Math. France, Paris, 1979, pp. 3-80. MR563472

[6] Jean-Marc Fontaine, Formes différentielles et modules de Tate des variétés abéliennes sur les corps locaux (French), Invent. Math. 65 (1981/82), no. 3, 379-409, DOI 10.1007/BF01396625 MR643559

[7] Jean-Marc Fontaine, Sur certains types de représentations p-adiques du groupe de Galois d'un corps local; construction d'un anneau de Barsotti-Tate (French), Ann. of Math. (2) 115 (1982), no. 3, 529-577, DOI 10.2307/2007012 MR657238

[8] Jean-Marc Fontaine, Cohomologie de de Rham, cohomologie cristalline et représentations p-adiques (French), Algebraic geometry (Tokyo/Kyoto, 1982), Lecture Notes in Math., vol. 1016, Springer, Berlin, 1983, pp. 86-108, DOI 10.1007/BFb0099959. MR726422

[9] Jean-Marc Fontaine, Il $n^{\prime} y$ a pas de variété abélienne sur $\mathbf{Z}$ (French), Invent. Math. 81 (1985), no. 3, 515-538, DOI 10.1007/BF01388584. MR807070

[10] Jean-Marc Fontaine and William Messing, p-adic periods and p-adic étale cohomology, Current trends in arithmetical algebraic geometry (Arcata, Calif., 1985), Contemp. Math., vol. 67, Amer. Math. Soc., Providence, RI, 1987, pp. 179207, DOI $10.1090 /$ conm/067/902593 MR902593

[11] Jean-Marc Fontaine, Letter to U. Jannsen, Nov. 26, 1987. 
[12] Jean-Marc Fontaine, Représentations p-adiques des corps locaux. I (French), The Grothendieck Festschrift, Vol. II, Progr. Math., vol. 87, Birkhäuser Boston, Boston, MA, 1990, pp. 249-309. MR1106901

[13] Jean-Marc Fontaine, Valeurs spéciales des fonctions $L$ des motifs (French, with French summary), Séminaire Bourbaki, Vol. 1991/92, Astérisque 206 (1992), Exp. No. 751, 4, 205-249. MR1206069

[14] Jean-Marc Fontaine, Le corps des périodes p-adiques (French), Périodes $p$-adiques (Bures-sur-Yvette, 1988), Astérisque 223 (1994), 59-111. With an appendix by Pierre Colmez. MR1293971

[15] Jean-Marc Fontaine, Représentations p-adiques semi-stables (French), Périodes p-adiques (Bures-sur-Yvette, 1988), Astérisque 223 (1994), 113-184. With an appendix by Pierre Colmez. MR1293972

[16] Jean-Marc Fontaine and Barry Mazur, Geometric Galois representations, Elliptic curves, modular forms, \& Fermat's last theorem (Hong Kong, 1993), Ser. Number Theory, I, Int. Press, Cambridge, MA, 1995, pp. 41-78. MR1363495

[17] Pierre Colmez and Jean-Marc Fontaine, Construction des représentations p-adiques semi-stables (French), Invent. Math. 140 (2000), no. 1, 1-43, DOI 10.1007/s002220000042. MR1779803

[18] Laurent Fargues and Jean-Marc Fontaine, Vector bundles and p-adic Galois representations, Fifth International Congress of Chinese Mathematicians. Part 1, 2, AMS/IP Stud. Adv. Math., 51, pt. 1, vol. 2, Amer. Math. Soc., Providence, RI, 2012, pp. 77-113. MR2908062

[19] Laurent Fargues and Jean-Marc Fontaine, Vector bundles on curves and p-adic Hodge theory, Automorphic forms and Galois representations. Vol. 2, London Math. Soc. Lecture Note Ser., vol. 415, Cambridge Univ. Press, Cambridge, 2014, pp. 17-104. MR3444231

[20] Laurent Fargues and Jean-Marc Fontaine, Courbes et fibrés vectoriels en théorie de Hodge p-adique (French, with English and French summaries), Astérisque 406 (2018), xiii+382. With a preface by Pierre Colmez. MR3917141

\section{Credits}

Figures 1 and 4 are courtesy of Isabelle Fontaine.

Figure 2 is courtesy of Gérard Laumon.

Figure 3 is courtesy of Pierre Berthelot.

Figures 5 and 6 are courtesy of the IHES/MCV.

Photo of Nicholas M. Katz is courtesy of Charles Mozzochi.

Photo of Peter Scholze is courtesy of the Archives of the Mathematisches Forschungsinstitut Oberwolfach.

The other author photos are courtesy of the authors.

\section{The Mathematical Society of Japan}

\section{Recent volumes from MSJ}

\section{Advanced Studies \\ in Pure Mathematics \\ http://mathsoc.jp/publication/ASPM/}

\section{Volume 84 \\ Various Aspects of \\ Multiple Zeta Functions \\ -- in honor of Professor \\ Kohji Matsumoto's \\ 60th birthday}

Edited by H. Mishou,

T. Nakamura, M. Suzuki,

Y. Umegaki

ISBN 978-4-86497-088-4

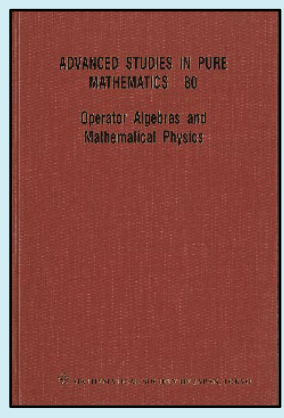

Volume 83

\section{Primitive Forms and Related Subjects} ---Kavli IPMU 2014

Edited by K. Hori, C. Li, S. Li, K. Saito

ISBN 978-4-86497-085-3

\section{MSJ Memoirs}

http://mathsoc.jp/publication/memoir/memoirs-e.html

Volume 38

Moduli spaces of real

projective structures

on surfaces

Alex Casella, Dominic Tate,

Stephan Tillmann

ISBN 978-4-86497-096-9

Volume 37

\section{4-dimensional}

\section{Painlevé-type equations}

Kazuki Hiroe, Hiroshi Kawakami,

Akane Nakamura, Hidetaka Sakai

ISBN 978-4-86497-087-7

$\nabla \nabla \nabla$ For purchase, visit $\nabla \nabla \nabla$

http://www.ams.org/bookstore/aspmseries

http://www.worldscientific.com/series/aspm

https://www.worldscientific.com/series/msjm

The Mathematical Society of Japan

34-8, Taito 1-chome, Taito-ku

Tokyo, JAPAN

www.mathsoc.jp/en/ 